\title{
Genetic Variation and Alleviation of Salinity Stress in Barley (Hordeum vulgare L.)
}

\author{
Mohamed A. El-Esawi ${ }^{1, * \mathbb{C}}$, Ibrahim A. Alaraidh ${ }^{2}$, Abdulaziz A. Alsahli ${ }^{2}$, Hayssam M. Ali ${ }^{2,3}$ (), \\ Aisha A. Alayafi ${ }^{4}$, Jacques Witczak ${ }^{5}$ and Margaret Ahmad ${ }^{5,6}$ \\ 1 Botany Department, Faculty of Science, Tanta University, Tanta 31527, Egypt \\ 2 Botany and Microbiology Department, College of Science, King Saud University, P.O. Box 2455, \\ Riyadh 11451, Saudi Arabia; ialaraidh@ksu.edu.sa (I.A.A.); aalshenaifi@ksu.edu.sa (A.A.A.); \\ hayhassan@ksu.edu.sa (H.M.A.) \\ 3 Timber Trees Research Department, Sabahia Horticulture Research Station, Horticulture Research Institute, \\ Agriculture Research Center, Alexandria 21526, Egypt \\ 4 Biological Sciences Department, Faculty of Science, University of Jeddah, Jeddah 21577, Saudi Arabia; \\ aal_shareaf@hotmail.com \\ 5 UMR CNRS 8256 (B2A), IBPS, Université Paris VI, 75005 Paris, France; jacques.witczak@upmc.fr (J.W.); \\ margaret.ahmad@upmc.fr (M.A.) \\ 6 Department of Biology, Xavier University, Cincinnati, OH 45207, USA \\ * Correspondence: mohamed.elesawi@science.tanta.edu.eg; Tel.: +20-40-3344352
}

Received: 7 September 2018; Accepted: 25 September 2018; Published: 28 September 2018

\begin{abstract}
Barley (Hordeum vulgare L.) represents one of the most important cereals cultivated worldwide. Investigating genetic variability and structure of barley is important for enhancing the crop productivity. This study aimed to investigate the diversity and structure of 40 barley genotypes originated from three European countries (France, the Netherlands, Poland) using amplified fragment length polymorphisms (AFLPs). It also aimed to study 5-aminolevulinic acid (ALA) effect on salinity tolerance of six barley genotypes. The expected heterozygosity $\left(H_{\mathrm{e}}\right)$ diverged from 0.126 to 0.501 , with a mean of 0.348. Polymorphic information content (PIC) diverged from 0.103 to 0.482 across barley genotypes, with a mean of 0.316 , indicating that barley genotypes are rich in a considerable level of genetic diversity. The 40 barley genotypes were further studied based on their geographical origin (Western Europe and Eastern Europe). The Eastern European region (Poland) has a higher barley variability than the Western European region (France and the Netherlands). Nei's distance-based cluster tree divided the 40 barley accessions into two major clusters; one cluster comprised all the varieties originated from the Eastern European region, while the other major cluster included all accessions originated from the Western European region. Structure analysis results were in a complete concordance with our cluster analysis results. Slaski 2, Damseaux and Urbanowicki genotypes have the highest diversity level, whereas Carmen, Bigo and Cambrinus genotypes have the lowest level. The response of these six varieties to $\mathrm{NaCl}$ stress was also investigated. Salt stress $(100 \mathrm{mM} \mathrm{NaCl})$ slightly decreased levels of chlorophyll, carotenoid and osmolytes (proteins, soluble sugars, phenolics and flavonoids) in the leaves of Slaski 2, Damseaux and Urbanowicki genotypes at non-significant level, as compared to control samples. However, pigment contents and osmolytes in leaves of Carmen, Bigo and Cambrinus genotypes were significantly decreased by salt stress. Antioxidant enzyme activities were significantly increased in Slaski 2 genotype, but non-significantly increased in Carmen by salt stress. Priming Slaski 2 and Carmen cultivars with ALA under salt stress significantly induced pigment contents, antioxidants enzymes activity and stress-responsive genes expression, relative to $\mathrm{NaCl}$-stressed plants. In conclusion, this study suggested a correlation between variability percentage and degree of salinity resistance. ALA improved salt tolerance in barley.
\end{abstract}

Keywords: AFLP; European barley; genetic diversity; population structure; salt tolerance; ALA 


\section{Introduction}

Barley (Hordeum vulgare L.) belongs to the major cereals grown in various environments worldwide [1,2]. It has a high economic value and is used in food and industry. Barley is adapted to different conditions and tolerant to several abiotic factors, including temperature, salinity, and water stress [2,3]. Cultivated barley and its wild progenitor are included in the primary gene pool [2,4]. Wild type has a higher allelic diversity than that of the cultivated one $[5,6]$. Wild type is also rich in genes that enable it to adapt to various abiotic and biotic factors [2,7]. Characterization and exploitation of such genes increase barley crop productivity and quality and augment crop breeding and conservation strategies.

Evaluation of genetic diversity levels and abiotic stress tolerance of crops is important for developing effective breeding programs to enhance crop productivity. Various methods have been applied to study the genetic variability and physiological mechanisms in plant species [8-29]. Over the last few years, different molecular traits including amplified fragment length polymorphisms (AFLPs), microsatellites (SSRs), diversity array technology (DArT) and inter simple sequence repeats (ISSRs) have been successfully utilized to study the variability for salinity tolerance in various plant species which would help identify salt tolerant genotypes and quantitative trait loci (QTLs) controlling salt tolerance for future exploitation in breeding programs to improve the crops and develop newly high salt tolerant varieties. For example, Krishnamurthy et al. [30] have successfully evaluated 94 rice varieties for salt tolerance using SSR markers located in the chromosome 1 Saltol QTL conferring salinity tolerance. The importance of SSR markers was also reported in studying Brassica breeding for salinity tolerance and identification of QTLs conferring salt tolerance [31]. Moreover, Kumar et al. [32] identified the variation in salinity tolerance in both Brassica and rice using SSR markers. Fan et al. [33] identified important QTLs conferring salt and drought tolerance using physiological and agronomic traits in barley population having a linkage map constructed based on several SSR, AFLP and DArT markers. Ahmadi-Ochtapeh et al. [34] also identified and characterized promising QTLs controlling salinity tolerance in barley population having a linkage genetic map constructed based on 106 SSR and AFLP markers.

Molecular makers have also been successfully used in detecting genetic variation levels and phylogenetic relationships in various plant species [35-37]. Moreover, genetic diversity and relationships have also been studied in barley germplasm collected from different geographical origins using morphological, biochemical, and molecular traits [2,38,39]. SDS-PAGE and isozyme analyses were used for studying the genetic diversity of Brazilian barley genotypes and showed diversity levels [40]. As morphological and biochemical characters might be influenced by surrounding environment, molecular traits have been therefore used to assess polymorphism and population structure. Random amplified polymorphic DNAs (RAPDs) were also used to evaluate the variability levels in barley [41]. Furthermore, AFLP and SSR markers proved useful for assessing barley diversity levels and structure due to their high polymorphism levels and exhibiting a considerable number of amplified fragments which are useful in diversity studies [2,42]. AFLPs and microsatellites have been successfully used to evaluate barley germplasm of various origins $[2,5,6,39,43,44]$. Those studies recorded different levels of diversity and provided useful information for understanding the diversity and relationships of barley; however most of them have analyzed a limited range of genotypes. Therefore, more information on genetic diversity, structure, and relationships of barley genotypes of different origins is still required. Genebanks and genetic resource centers maintain barley germplasm that need further genetic characterization to exploit their genetic diversity and tolerance to abiotic stresses in breeding programs for enhancing the crop quality and yield. The objectives of the current investigation were therefore to study variability level, structure, and relationships of 40 barley genotypes originated from three European countries (France, the Netherlands, Poland) using AFLP markers. Additionally, this study aimed at investigating the correlation between levels of diversity and salinity $(\mathrm{NaCl})$ tolerance in some cultivars contrasting in their genetic diversity levels, and evaluated the 5-aminolevulinic acid (ALA) growth regulator effects on salt tolerance in those cultivars. 


\section{Results and Discussion}

\subsection{Molecular Diversity Analysis in Barley Genotypes}

\subsubsection{Polymorphism Analysis and Diversity Indices of AFLP Markers}

Several methods have been applied in assessing genetic diversity levels in crops to enhance their productivity and quality. Because morphological traits are influenced by environmental conditions, molecular markers have been efficiently utilized in barely variability studies. AFLP proved to be a powerful technique for cultivar identification characterization. The current study used AFLP markers to characterize the molecular variability and structure of barley varieties originated from three countries (France, the Netherlands, and Poland). Across the 14 AFLP primer sets used, 760 AFLP bands were recorded in the 160 individuals of the 40 varieties studied (Table 1). The AFLP fragments varied in molecular size from 45 to $785 \mathrm{bp}$, and in number from 28 to 75 with an average of 54.29 (Table 1). Primer pair (E-AGG/M-CAG) revealed the lowest fragment number (28), with molecular sizes varying from 55 to $490 \mathrm{bp}$ (Table 1). The primer set (E-ACC/M-CAG) revealed the highest fragment number (75), with molecular sizes varying from 75 to $780 \mathrm{bp}$. All the 14 AFLP primer sets were polymorphic. Polymorphic fragments diverged in number from 18 (E-AAG/M-CTT) to 52 (E-AGG/M-CTA) with a mean of 35.57. This mean was lower than that recorded in barley (58.5) by Assefa et al. [45], but higher than that recorded by Varshney et al. [46] for barley (27). Furthermore, the polymorphism percentage varied from $45.33 \%$ (E-ACC/M-CAG) to $92.73 \%$ (E-ACT/M-CAG), with an average of $66.07 \%$ (Table 1). This mean polymorphism percentage was relatively similar to that reported by Assefa et al. [45], but higher than that recorded by Adawy et al. [44]. The gene diversity of AFLP primer sets diverged from 0.17 (E-AAG/M-CTG) to 0.48 (E-ACT/M-CAG), with an average of 0.37 , indicating a good level of genetic diversity within barely genotypes. Polymorphic information content (PIC) exhibited a mean of 0.33 (Table 1). This mean was higher than that indicated by Varshney et al. [46]. The difference in this data is a result of the differences in the barely genotypes analyzed, and molecular markers used. The AFLP primer sets revealing a high degree of polymorphism in the present study are recommended for use in future diversity studies of barley germplasm.

Table 1. AFLP primers, fragments number and genetic variability indices in the 40 barely varieties.

\begin{tabular}{ccccccc}
\hline AFLP Primer Sets & $\begin{array}{c}\text { Number of } \\
\text { Fragments }\end{array}$ & $\begin{array}{c}\text { No. of } \\
\text { Polymorphic } \\
\text { Fragments }\end{array}$ & $\begin{array}{c}\text { Polymorphism } \\
\mathbf{( \% )}\end{array}$ & $\begin{array}{c}\text { Fragments } \\
\text { Size Range }\end{array}$ & $\begin{array}{c}\text { Gene } \\
\text { Diversity }\end{array}$ & PIC \\
\hline E-AAC/M-CTT & 31 & 22 & 71.01 & $55-220$ & 0.45 & 0.41 \\
E-ACT/M-CTG & 49 & 31 & 63.27 & $50-560$ & 0.41 & 0.36 \\
E-ACA/M-CAG & 42 & 21 & 50.00 & $95-520$ & 0.32 & 0.29 \\
E-ACT/M-CAG & 55 & 51 & 92.73 & $75-490$ & 0.48 & 0.44 \\
E-AGG/M-CTA & 69 & 52 & 75.36 & $90-785$ & 0.35 & 0.32 \\
E-AAG/M-CTT & 31 & 18 & 58.06 & $95-610$ & 0.44 & 0.41 \\
E-ACT/M-CTT & 62 & 43 & 69.36 & $50-520$ & 0.42 & 0.38 \\
E-ACC/M-CTA & 44 & 34 & 77.27 & $60-540$ & 0.45 & 0.42 \\
E-AGG/M-CAG & 28 & 19 & 67.86 & $55-490$ & 0.39 & 0.36 \\
E-AAG/M-CTG & 72 & 41 & 56.94 & $45-540$ & 0.17 & 0.14 \\
E-AGG/M-CAC & 68 & 51 & 75.00 & $60-560$ & 0.44 & 0.39 \\
E-ACC/M-CAC & 73 & 37 & 50.68 & $55-750$ & 0.22 & 0.19 \\
E-AGG/M-CTT & 61 & 44 & 72.13 & $65-490$ & 0.43 & 0.39 \\
E-ACC/M-CAG & 75 & 34 & 45.33 & $75-780$ & 0.19 & 0.15 \\
Mean & 54.29 & 35.57 & 66.07 & - & 0.37 & 0.33 \\
\hline
\end{tabular}

M, 5'-GATGAGTCCTGAGTAA-3'; E, 5'-GACTGCGTACCAATTC-3'; PIC, polymorphic information content. 


\subsubsection{Genetic Diversity of Barley Genotypes}

Table 2 exhibits the genetic variability indices calculated for each of the 40 barley genotypes. Observed heterozygosity $\left(H_{\mathrm{o}}\right)$ diverged from 0.127 (variety Chevalier from France) to 0.374 (Slaski 2 from Poland). Expected heterozygosity $\left(H_{\mathrm{e}}\right)$ diverged from 0.126 (Carmen from France) to 0.501 (Slaski 2 from Poland), with a mean of 0.348. PIC also diverged from 0.103 (Carmen from France) to 0.482 (Slaski 2 from Poland), with a mean of 0.316. These results indicate that Carmen cultivar comprised the lowest diversity level, whereas Slaski 2 comprised the highest diversity level. Three accessions (Damseaux from France, Urbanowicki from Poland, and Slaski 2 from Poland) exhibited negative fixation indices $(F)$ (Table 2), indicating the presence of higher level of heterozygosity in those accessions.

Table 2. Genetic variability indices of the 40 barley accessions analyzed.

\begin{tabular}{|c|c|c|c|c|c|c|}
\hline Accession Number & Cultivar Name & Origin & $H_{\mathrm{o}}$ & $H_{\mathrm{e}}$ & $F$ & PIC \\
\hline CGN00328 & Albert & France & 0.312 & 0.415 & 0.301 & 0.381 \\
\hline CGN02709 & Ares & France & 0.325 & 0.391 & 0.214 & 0.362 \\
\hline CGN02712 & Astrix & France & 0.213 & 0.289 & 0.211 & 0.244 \\
\hline CGN00329 & Baronne & France & 0.332 & 0.428 & 0.282 & 0.392 \\
\hline CGN11185 & Beatrice & France & 0.274 & 0.337 & 0.182 & 0.298 \\
\hline CGN00350 & Berenice & France & 0.261 & 0.368 & 0.298 & 0.331 \\
\hline CGN00330 & Berrichonne & France & 0.318 & 0.411 & 0.305 & 0.379 \\
\hline CGN00318 & Betina & France & 0.217 & 0.299 & 0.245 & 0.262 \\
\hline CGN00351 & Carmen & France & 0.131 & 0.126 & 0.041 & 0.103 \\
\hline CGN00339 & Ceres & France & 0.253 & 0.326 & 0.212 & 0.291 \\
\hline CGN00337 & Chevalier & France & 0.127 & 0.226 & 0.303 & 0.203 \\
\hline CGN00325 & Comtesse & France & 0.247 & 0.357 & 0.332 & 0.317 \\
\hline CGN21737 & Cytris & France & 0.317 & 0.431 & 0.368 & 0.391 \\
\hline CGN02043 & Damseaux & France & 0.286 & 0.469 & -0.537 & 0.481 \\
\hline CGN16158 & Flamenco & France & 0.216 & 0.325 & 0.336 & 0.292 \\
\hline CGN23651 & Gerbel & France & 0.292 & 0.427 & 0.392 & 0.391 \\
\hline CGN00008 & Agio & Netherlands & 0.263 & 0.352 & 0.274 & 0.312 \\
\hline CGN19325 & Anoa & Netherlands & 0.165 & 0.257 & 0.278 & 0.223 \\
\hline CGN21734 & Apex & Netherlands & 0.262 & 0.324 & 0.151 & 0.281 \\
\hline CGN21735 & Bellona & Netherlands & 0.236 & 0.326 & 0.274 & 0.285 \\
\hline CGN00003 & Bigo & Netherlands & 0.142 & 0.206 & 0.246 & 0.104 \\
\hline CGN00016 & Cambrinus & Netherlands & 0.221 & 0.207 & 0.031 & 0.106 \\
\hline CGN19319 & Cumbia & Netherlands & 0.142 & 0.235 & 0.314 & 0.192 \\
\hline CGN00014 & Delta & Netherlands & 0.253 & 0.341 & 0.274 & 0.298 \\
\hline CGN19330 & Efron & Netherlands & 0.267 & 0.363 & 0.302 & 0.325 \\
\hline CGN00230 & Germania & Netherlands & 0.273 & 0.327 & 0.151 & 0.284 \\
\hline CGN19326 & Grosso & Netherlands & 0.287 & 0.364 & 0.241 & 0.328 \\
\hline CGN08532 & Hexa & Netherlands & 0.291 & 0.425 & 0.361 & 0.385 \\
\hline CGN00450 & Antalek & Poland & 0.353 & 0.442 & 0.271 & 0.478 \\
\hline CGN00449 & Damazy & Poland & 0.226 & 0.389 & 0.392 & 0.449 \\
\hline CGN00385 & Danzig & Poland & 0.253 & 0.432 & 0.461 & 0.398 \\
\hline CGN00407 & Groesjetski & Poland & 0.297 & 0.454 & 0.411 & 0.413 \\
\hline CGN02119 & Kujawiak 2 & Poland & 0.184 & 0.306 & 0.352 & 0.299 \\
\hline CGN02106 & Kujawiak & Poland & 0.288 & 0.437 & 0.373 & 0.391 \\
\hline CGN00451 & Lubuski & Poland & 0.198 & 0.328 & 0.342 & 0.284 \\
\hline CGN00448 & Skreszowicki & Poland & 0.349 & 0.242 & 0.031 & 0.311 \\
\hline CGN02107 & Slaski 1 & Poland & 0.185 & 0.278 & 0.272 & 0.279 \\
\hline CGN02109 & Slaski 2 & Poland & 0.374 & 0.501 & -0.393 & 0.482 \\
\hline CGN02118 & Urbanowicki & Poland & 0.311 & 0.463 & -0.411 & 0.480 \\
\hline CGN02103 & W.R.112 Grodkowicki & Poland & 0.187 & 0.278 & 0.273 & 0.233 \\
\hline Mean & & & 0.253 & 0.348 & 0.281 & 0.311 \\
\hline
\end{tabular}

$H_{\mathrm{o}}$, observed heterozygosity; $F$, fixation index; $H_{\mathrm{e}}$, expected heterozygosity; PIC, polymorphic information content. 
Moreover, the 40 barley genotypes studied were split into 2 groups based on their geographical location (Western Europe and Eastern Europe). Western Europe includes the accessions originated from France and the Netherlands, while Eastern Europe includes the accessions originated from Poland. Table 3 shows the diversity indices calculated for the barley genotypes of Western and Eastern European regions. The observed heterozygosity $\left(H_{\mathrm{o}}\right)$ of the Western and Eastern European regions was 0.247 and 0.267 , respectively. The expected heterozygosity $\left(H_{\mathrm{e}}\right)$ of the Western and Eastern European regions was 0.335 and 0.380 , respectively. The fixation index of the Western and Eastern European regions was 0.261 and 0.327, respectively. PIC value of the Western and Eastern European regions was 0.298 and 0.368 , respectively. These diversity indices results indicate that the Eastern European region (Poland) has a higher barley variability than the Western European region (France and Netherlands). Therefore, barley genotypes originated from Poland could be further exploited to improve the crop.

Table 3. Genetic variability indices of barley accessions in the two European regions.

\begin{tabular}{cccccc}
\cline { 2 - 5 } & Barley Group & $\boldsymbol{H}_{\mathbf{o}}$ & $\boldsymbol{H}_{\mathbf{e}}$ & $\boldsymbol{F}$ & $\boldsymbol{P I C}$ \\
\cline { 2 - 6 } Western Europe & 0.247 & 0.335 & 0.261 & 0.298 \\
Eastern Europe & 0.267 & 0.380 & 0.327 & 0.368 \\
\hline$H_{\mathrm{o}}$, observed heterozygosity; $F$, fixation index; $H_{\mathrm{e}}$, expected heterozygosity; $P I C$, polymorphic. information content.
\end{tabular}

\subsubsection{Cluster Analysis and Population Structure of Barley Genotypes}

Nei's distance-based cluster tree was performed using AFLP data and exhibited the relationships among the 40 barley accessions studied (Figure 1). The dendrogram split into 2 major clusters; one cluster had all the 12 barley accessions originated from the Eastern European region (Poland), while the other cluster included all the barley accessions originated from the Western European region (France and the Netherlands) and split into two sub-clusters. The first one included 11 out of the 12 accessions originated from the Netherlands (Agio, Bigo, Delta, Hexa, Bellona, Cumbia, Germania, Apex, Cambrinus, Grosso, and Efron) and three accessions from France (Flamenco, Cytris, Gerbel). The second sub-cluster comprised one accession originated from the Netherlands (Anoa) and the remaining 13 accessions originated from France (Damseaux, Comtesse, Chevalier, Ceres, Carmen, Betina, Berrichonne, Berenice, Beatrice, Baronne, Astrix, Ares, Albert). The cluster analysis results indicate that barley accessions originated from the Western European region (France and the Netherlands) were more closely related to each other than to that originated from Eastern Europe (Poland).

Genetic structure of the 40 barley accessions was studied using the software STRUCTURE 2.3. This genetic analysis can estimate the hypothetical populations number to which barley accessions should be assigned. Structure analysis of barely genotypes revealed that $K=2$ had the highest $\Delta K$ value (Figure 2). Additionally, the fourth run was the best among the ten runs for $K=2$ based on the likelihood values. Consequently, $K=2$ represented the genetic structure of barely accessions studied, indicating that the 40 barely accessions could be assigned to two populations. At $K=2$, all barely genotypes originated from Western Europe (16 genotypes from France and 12 genotypes from the Netherlands) were assigned to one population (Pop. A) (Figure 1), while all genotypes from the Eastern European region (12 genotypes from Poland) were included the second population (Pop. B). The genetic structure results were in harmony with our cluster analysis data, confirming that barley accessions originated from Western Europe (France and the Netherlands) were more closely related to each other than to that originated from Eastern Europe (Poland). 


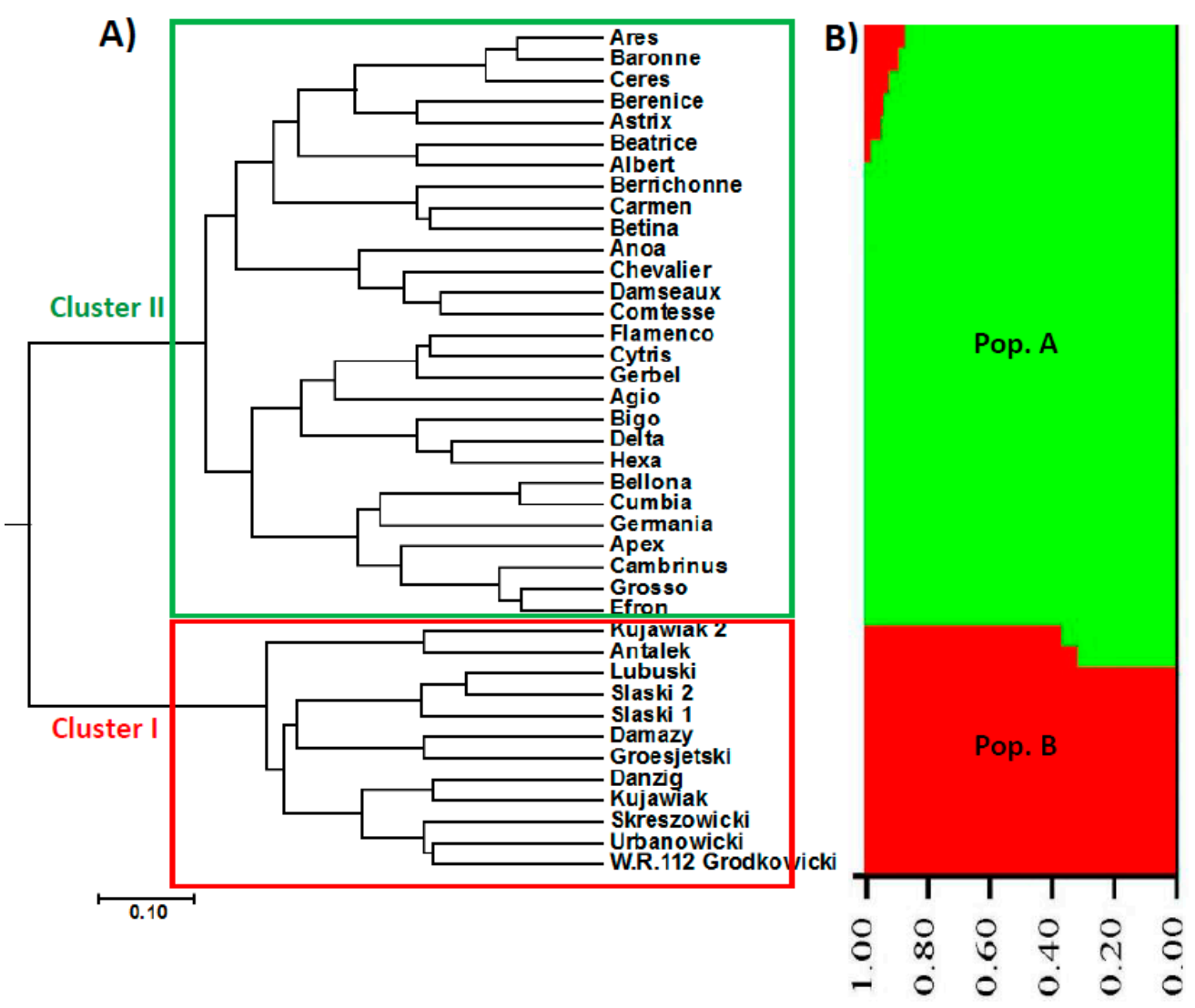

Figure 1. UPGMA phylogenetic tree and population structure (at $K=2$ ) of the 40 barley accessions based on AFLP data; (A) Cluster I included the Eastern European genotypes (Poland), while Cluster II represents the Western European genotypes (France and the Netherlands). (B) Structure analysis showing that the 40 barely accessions are assigned to two populations (Pop. A represented in green comprised the Western European genotypes, while Pop. B represented in red included the Eastern European genotypes).

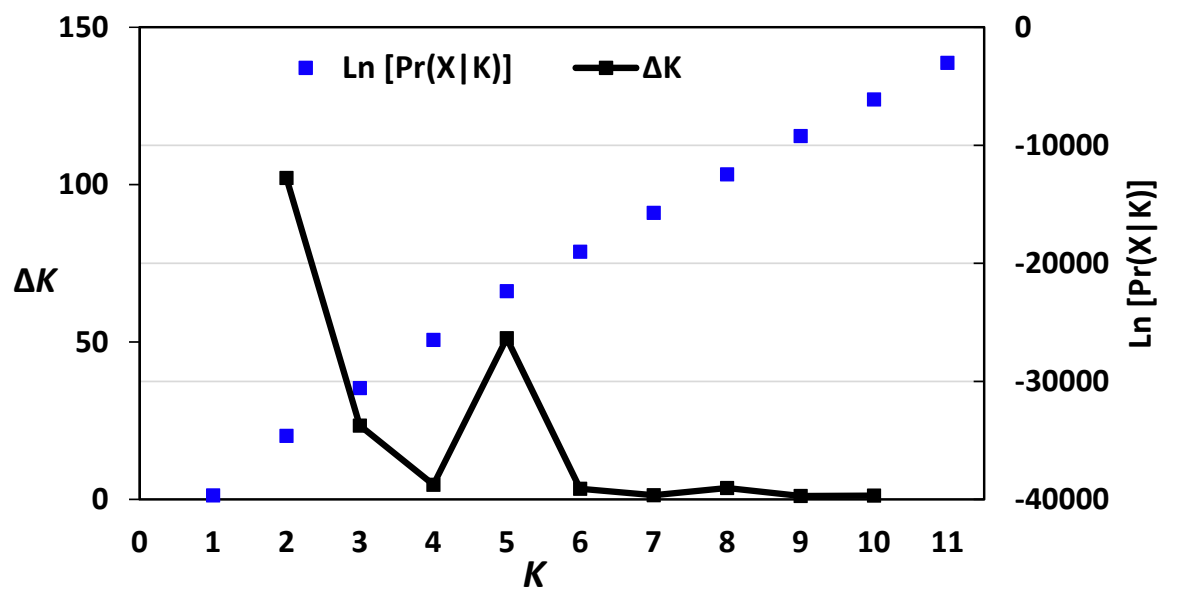

Figure 2. Log-likelihood and $\Delta K$ values of structure analysis of the 40 barley accessions based on AFLP data. $K=2$ had the highest $\Delta K$ value, indicating that the 40 barely accessions could be assigned to two populations.

\subsection{5-Aminolevulinic Acid Effect on Salt Tolerance in Barley}

Our AFLP results showed that Slaski 2, Damseaux and Urbanowicki genotypes contained the highest genetic diversity level, whereas Carmen, Bigo and Cambrinus genotypes comprised the lowest diversity level (Table 2). We, Therefore, chose these six variable genotypes to study their 
responsiveness to salt stress and evaluate whether correlation between their variability percentage and salinity tolerance level exists. Effects of 5-aminolevulinic acid on the tolerance of those six genotypes to salt stress were also studied.

The salt stress $(100 \mathrm{mM} \mathrm{NaCl})$ slightly decreased levels of chlorophyll, carotenoids and osmolytes (proteins, soluble sugars, phenolics and flavonoids) in the leaves of Slaski 2, Damseaux and Urbanowicki genotypes at non-significant level, as compared to controls (Tables 4-6). Moreover, salt stress slightly increased malondialdehyde (MDA) and hydrogen peroxide levels in leaves of Slaski 2, Damseaux and Urbanowicki genotypes. Treating the salt-stressed Slaski 2, Damseaux and Urbanowicki genotypes with ALA significantly induced the biosynthesis of pigments and osmolytes but significantly reduced the levels of hydrogen peroxide and MDA, relative to the plants treated with $\mathrm{NaCl}$ alone (Tables 4-6). Furthermore, priming the non-stressed Slaski 2, Damseaux and Urbanowicki genotypes with ALA significantly augmented the synthesis of pigments and osmolytes but reduced the levels of hydrogen peroxide and MDA, as compared to the untreated control plants. ALA enhanced proline accumulation and the antioxidant activity (DPPH) in the three salt-stressed genotypes (Tables 4-6).

Table 4. Contents of chlorophyll, carotenoids, proteins, sugars, proline, phenolics, flavonoids, $\mathrm{H}_{2} \mathrm{O}_{2}$, malondialdehyde (MDA) and antioxidant activity (DPPH) in leaves of Slaski 2 cultivar under salt and/or ALA (7 ppm) treatments.

\begin{tabular}{|c|c|c|c|c|}
\hline \multirow{2}{*}{$\mathrm{NaCl}$ Treatment } & \multicolumn{2}{|c|}{$0 \mathrm{mM}$} & \multicolumn{2}{|c|}{$100 \mathrm{mM}$} \\
\hline & -ALA & + ALA & -ALA & $+\mathrm{ALA}$ \\
\hline Chlorophyll a (mg.g $\left.{ }^{-1} \mathrm{FW}\right)$ & $2.71 \pm 0.13^{c}$ & $2.98 \pm 0.15^{\mathrm{a}}$ & $2.59 \pm 0.11^{\mathrm{c}}$ & $2.80 \pm 0.13^{b}$ \\
\hline Chlorophyll b (mg.g $\left.{ }^{-1} \mathrm{FW}\right)$ & $1.39 \pm 0.06^{c}$ & $1.71 \pm 0.06^{\mathrm{a}}$ & $1.33 \pm 0.04^{\mathrm{c}}$ & $1.51 \pm 0.07^{b}$ \\
\hline Total Chl $\left(\mathrm{mg} \cdot \mathrm{g}^{-1} \mathrm{FW}\right)$ & $4.10 \pm 0.12^{c}$ & $4.69 \pm 0.14^{\mathrm{a}}$ & $3.99 \pm 0.17^{c}$ & $4.31 \pm 0.14^{b}$ \\
\hline Carotenoid $\left(\mathrm{mg} \cdot \mathrm{g}^{-1} \mathrm{FW}\right)$ & $0.32 \pm 0.06^{c}$ & $0.37 \pm 0.07^{\mathrm{a}}$ & $0.30 \pm 0.09^{c}$ & $0.34 \pm 0.06^{b}$ \\
\hline Proteins $\left(\mathrm{mg} \cdot \mathrm{g}^{-1} \mathrm{FW}\right)$ & $0.92 \pm 0.11^{\mathrm{c}}$ & $1.07 \pm 0.07^{\mathrm{a}}$ & $0.86 \pm 0.08^{c}$ & $0.98 \pm 0.09^{b}$ \\
\hline Soluble sugars $\left(\mu \mathrm{g} \cdot \mathrm{g}^{-1} \mathrm{FW}\right)$ & $2.12 \pm 0.16^{\mathrm{c}}$ & $2.35 \pm 0.12^{\mathrm{a}}$ & $2.04 \pm 0.14^{\mathrm{c}}$ & $2.19 \pm 0.17^{b}$ \\
\hline Proline $\left(\mu \mathrm{g} \cdot \mathrm{g}^{-1} \mathrm{FW}\right)$ & $14.4 \pm 0.47^{\mathrm{d}}$ & $21.8 \pm 0.51^{c}$ & $25.9 \pm 0.42^{b}$ & $30.8 \pm 0.51^{\mathrm{a}}$ \\
\hline Total Phenolics $\left(\mu \mathrm{mol} \cdot \mathrm{g}^{-1} \mathrm{FW}\right)$ & $14.3 \pm 0.33^{c}$ & $16.8 \pm 0.41^{\mathrm{a}}$ & $13.1 \pm 0.31^{\mathrm{c}}$ & $14.8 \pm 0.32^{b}$ \\
\hline Total Flavonoids $\left(\mu \mathrm{mol} \cdot \mathrm{g}^{-1} \mathrm{FW}\right)$ & $7.11 \pm 0.18^{c}$ & $8.89 \pm 0.15^{a}$ & $6.41 \pm 0.17^{c}$ & $7.22 \pm 0.13^{b}$ \\
\hline $\mathrm{H}_{2} \mathrm{O}_{2}\left(\mu \mathrm{mol} \cdot \mathrm{g}^{-1} \mathrm{FW}\right)$ & $0.23 \pm 0.03^{c}$ & $0.18 \pm 0.05^{\mathrm{d}}$ & $0.34 \pm 0.04^{\mathrm{a}}$ & $0.29 \pm 0.06^{b}$ \\
\hline $\mathrm{MDA}\left(\mu \mathrm{mol} \cdot \mathrm{g}^{-1} \mathrm{FW}\right)$ & $0.17 \pm 0.05^{c}$ & $0.15 \pm 0.04^{\mathrm{d}}$ & $0.28 \pm 0.03^{a}$ & $0.23 \pm 0.05^{b}$ \\
\hline $\mathrm{DPPH}\left(\mathrm{IC}_{50}, \mu \mathrm{g} \cdot \mathrm{mL}^{-1}\right)$ & $0.35 \pm 0.02^{a}$ & $0.32 \pm 0.04^{c}$ & $0.36 \pm 0.02^{b}$ & $0.31 \pm 0.02^{\mathrm{d}}$ \\
\hline
\end{tabular}

Different alphabetical letters presented in the same row refer to significant differences among the applied treatments $(p \leq 0.05)$.

Table 5. Contents of chlorophylls, carotenoids, proteins, sugars, proline, phenolics, flavonoids, $\mathrm{H}_{2} \mathrm{O}_{2}$, MDA and antioxidant activity (DPPH) in leaves of Damseaux cultivar under salt and/or ALA (7 ppm) treatments.

\begin{tabular}{|c|c|c|c|c|}
\hline \multirow{2}{*}{$\mathrm{NaCl}$ Treatment } & \multicolumn{2}{|c|}{$0 \mathrm{mM}$} & \multicolumn{2}{|c|}{$100 \mathrm{mM}$} \\
\hline & - ALA & + ALA & - ALA & + ALA \\
\hline Chlorophyll a (mg.g $\left.{ }^{-1} \mathrm{FW}\right)$ & $2.67 \pm 0.14^{\mathrm{c}}$ & $2.93 \pm 0.13^{\mathrm{a}}$ & $2.59 \pm 0.13^{c}$ & $2.81 \pm 0.14^{b}$ \\
\hline Chlorophyll b (mg $\left.\cdot \mathrm{g}^{-1} \mathrm{FW}\right)$ & $1.41 \pm 0.07^{c}$ & $1.69 \pm 0.05^{\mathrm{a}}$ & $1.33 \pm 0.06^{c}$ & $1.48 \pm 0.05^{b}$ \\
\hline Total Chl $\left(\mathrm{mg} \cdot \mathrm{g}^{-1} \mathrm{FW}\right)$ & $4.08 \pm 0.14^{c}$ & $4.62 \pm 0.11^{\mathrm{a}}$ & $3.98 \pm 0.13^{c}$ & $4.29 \pm 0.13^{b}$ \\
\hline Carotenoid $\left(\mathrm{mg} \cdot \mathrm{g}^{-1} \mathrm{FW}\right)$ & $0.32 \pm 0.05^{c}$ & $0.41 \pm 0.04^{\mathrm{a}}$ & $0.29 \pm 0.07^{c}$ & $0.35 \pm 0.04^{b}$ \\
\hline Proteins $\left(\mathrm{mg} \cdot \mathrm{g}^{-1} \mathrm{FW}\right)$ & $0.86 \pm 0.08^{c}$ & $1.11 \pm 0.09^{\mathrm{a}}$ & $0.82 \pm 0.07^{\mathrm{c}}$ & $1.03 \pm 0.07^{b}$ \\
\hline Soluble sugars $\left(\mu \mathrm{g} \cdot \mathrm{g}^{-1} \mathrm{FW}\right)$ & $2.07 \pm 0.12^{\mathrm{c}}$ & $2.27 \pm 0.11^{\mathrm{a}}$ & $2.02 \pm 0.15^{c}$ & $2.17 \pm 0.13^{b}$ \\
\hline Proline $\left(\mu \mathrm{g} \cdot \mathrm{g}^{-1} \mathrm{FW}\right)$ & $14.1 \pm 0.39^{d}$ & $19.2 \pm 0.41^{c}$ & $24.3 \pm 0.37^{b}$ & $32.2 \pm 0.43^{a}$ \\
\hline Total Phenolics $\left(\mu \mathrm{mol} \cdot \mathrm{g}^{-1} \mathrm{FW}\right)$ & $13.6 \pm 0.22^{c}$ & $14.9 \pm 0.27^{\mathrm{a}}$ & $12.9 \pm 0.29^{c}$ & $14.1 \pm 0.27^{\mathrm{b}}$ \\
\hline Total Flavonoids $\left(\mu \mathrm{mol} \cdot \mathrm{g}^{-1} \mathrm{FW}\right)$ & $6.57 \pm 0.13^{c}$ & $7.97 \pm 0.11^{\mathrm{a}}$ & $5.98 \pm 0.15^{c}$ & $6.99 \pm 0.12^{b}$ \\
\hline $\mathrm{H}_{2} \mathrm{O}_{2}\left(\mu \mathrm{mol} \cdot \mathrm{g}^{-1} \mathrm{FW}\right)$ & $0.21 \pm 0.05^{c}$ & $0.17 \pm 0.04^{\mathrm{d}}$ & $0.31 \pm 0.06^{\mathrm{a}}$ & $0.26 \pm 0.07^{b}$ \\
\hline MDA $\left(\mu \mathrm{mol} \cdot \mathrm{g}^{-1} \mathrm{FW}\right)$ & $0.15 \pm 0.03^{c}$ & $0.13 \pm 0.05^{\mathrm{d}}$ & $0.26 \pm 0.06^{\mathrm{a}}$ & $0.21 \pm 0.05^{b}$ \\
\hline $\mathrm{DPPH}\left(\mathrm{IC}_{50}, \mu \mathrm{g} \cdot \mathrm{mL}^{-1}\right)$ & $0.33 \pm 0.03^{a}$ & $0.31 \pm 0.05^{\mathrm{c}}$ & $0.34 \pm 0.03^{b}$ & $0.29 \pm 0.04^{\mathrm{d}}$ \\
\hline
\end{tabular}

Different alphabetical letters in the same row show significant differences among the applied treatments $(p \leq 0.05)$. 
Table 6. Contents of chlorophyll, carotenoids, proteins, sugars, proline, phenolics, flavonoids, $\mathrm{H}_{2} \mathrm{O}_{2}$, MDA and antioxidant activity (DPPH) in leaves of Urbanowicki cultivar under salt and/or ALA (7 ppm) treatments.

\begin{tabular}{|c|c|c|c|c|}
\hline \multirow{2}{*}{$\mathrm{NaCl}$ Treatment } & \multicolumn{2}{|c|}{$0 \mathrm{mM}$} & \multicolumn{2}{|c|}{$100 \mathrm{mM}$} \\
\hline & $-\mathbf{A L A}$ & + ALA & $-\mathrm{ALA}$ & $+\mathrm{ALA}$ \\
\hline Chlorophyll a $\left(\mathrm{mg} \cdot \mathrm{g}^{-1} \mathrm{FW}\right)$ & $2.69 \pm 0.11^{\mathrm{c}}$ & $2.88 \pm 0.15^{a}$ & $2.57 \pm 0.12^{\mathrm{c}}$ & $2.74 \pm 0.13^{b}$ \\
\hline Chlorophyll b $\left(\mathrm{mg} \cdot \mathrm{g}^{-1} \mathrm{FW}\right)$ & $1.39 \pm 0.04^{\mathrm{c}}$ & $1.58 \pm 0.06^{\mathrm{a}}$ & $1.32 \pm 0.05^{\mathrm{c}}$ & $1.41 \pm 0.04^{b}$ \\
\hline Total Chl $\left(\mathrm{mg} \cdot \mathrm{g}^{-1} \mathrm{FW}\right)$ & $4.08 \pm 0.13^{c}$ & $4.46 \pm 0.14^{\mathrm{a}}$ & $3.96 \pm 0.12^{\mathrm{c}}$ & $4.15 \pm 0.11^{b}$ \\
\hline Carotenoid $\left(\mathrm{mg} \cdot \mathrm{g}^{-1} \mathrm{FW}\right)$ & $0.31 \pm 0.06^{\mathrm{c}}$ & $0.38 \pm 0.05^{\mathrm{a}}$ & $0.29 \pm 0.06^{c}$ & $0.37 \pm 0.07^{b}$ \\
\hline Proteins $\left(\mathrm{mg} \cdot \mathrm{g}^{-1} \mathrm{FW}\right)$ & $0.91 \pm 0.07^{\mathrm{c}}$ & $1.22 \pm 0.08^{a}$ & $0.85 \pm 0.08^{\mathrm{c}}$ & $1.07 \pm 0.06^{b}$ \\
\hline Soluble sugars $\left(\mu \mathrm{g} \cdot \mathrm{g}^{-1} \mathrm{FW}\right)$ & $2.03 \pm 0.11^{\mathrm{c}}$ & $2.18 \pm 0.13^{a}$ & $1.98 \pm 0.12^{\mathrm{c}}$ & $2.08 \pm 0.11^{b}$ \\
\hline Proline $\left(\mu \mathrm{g} \cdot \mathrm{g}^{-1} \mathrm{FW}\right)$ & $13.9 \pm 0.29^{d}$ & $18.3 \pm 0.38^{c}$ & $25.7 \pm 0.27^{b}$ & $31.8 \pm 0.34^{\mathrm{a}}$ \\
\hline Total Phenolics $\left(\mu \mathrm{mol} \cdot \mathrm{g}^{-1} \mathrm{FW}\right)$ & $13.8 \pm 0.21^{\mathrm{c}}$ & $14.6 \pm 0.22^{a}$ & $12.9 \pm 0.25^{c}$ & $14.1 \pm 0.23^{b}$ \\
\hline Total Flavonoids $\left(\mu \mathrm{mol} \cdot \mathrm{g}^{-1} \mathrm{FW}\right)$ & $7.05 \pm 0.13^{c}$ & $7.86 \pm 0.12^{a}$ & $5.98 \pm 0.11^{\mathrm{c}}$ & $7.02 \pm 0.13^{b}$ \\
\hline $\mathrm{H}_{2} \mathrm{O}_{2}\left(\mu \mathrm{mol} \cdot \mathrm{g}^{-1} \mathrm{FW}\right)$ & $0.19 \pm 0.06^{\mathrm{c}}$ & $0.16 \pm 0.03^{d}$ & $0.28 \pm 0.08^{a}$ & $0.23 \pm 0.05^{b}$ \\
\hline $\mathrm{MDA}\left(\mu \mathrm{mol} \cdot \mathrm{g}^{-1} \mathrm{FW}\right)$ & $0.14 \pm 0.05^{c}$ & $0.11 \pm 0.03^{d}$ & $0.24 \pm 0.07^{\mathrm{a}}$ & $0.19 \pm 0.06^{b}$ \\
\hline $\mathrm{DPPH}\left(\mathrm{IC}_{50}, \mu \mathrm{g} \cdot \mathrm{mL}^{-1}\right)$ & $0.31 \pm 0.04^{\mathrm{a}}$ & $0.29 \pm 0.04^{b}$ & $0.32 \pm 0.02^{c}$ & $0.30 \pm 0.03^{d}$ \\
\hline
\end{tabular}

Different alphabetical letters in the same row show significant differences among the applied treatments $(p \leq 0.05)$.

On the other hand, salt stress $(100 \mathrm{mM} \mathrm{NaCl})$ significantly decreased the contents of photosynthetic pigments and osmolytes in the leaves of Carmen, Bigo and Cambrinus genotypes, as compared to the untreated control plants (Tables 7-9). Moreover, $\mathrm{NaCl}$ stress significantly enhanced hydrogen peroxide and MDA contents in leaves of Carmen, Bigo and Cambrinus genotypes. Treating the salt-stressed Carmen, Bigo and Cambrinus genotypes with ALA significantly induced pigments biosynthesis and osmolytes contents but significantly reduced the levels of hydrogen peroxide and MDA, relative to the plants treated with salt alone (Tables 7-9). Furthermore, priming the control Carmen, Bigo and Cambrinus plants with ALA significantly induced photosynthetic pigments biosynthesis and osmolytes contents but reduced the levels of hydrogen peroxide and malondialdehyde, as compared to the untreated control plants (Tables 7-9). Moreover, ALA treatment enhanced proline biosynthesis and antioxidant activity (DPPH) in those three salinity-stressed barley genotypes (Tables 7-9).

Table 7. Contents of chlorophyll, carotenoids, proteins, sugars, proline, phenolics, flavonoids, $\mathrm{H}_{2} \mathrm{O}_{2}$, MDA and antioxidant activity (DPPH) in leaves of Carmen cultivar under salt and/or ALA (7 ppm) treatments.

\begin{tabular}{|c|c|c|c|c|}
\hline \multirow{2}{*}{$\mathrm{NaCl}$ Treatment } & \multicolumn{2}{|c|}{$0 \mathrm{mM}$} & \multicolumn{2}{|c|}{$100 \mathrm{mM}$} \\
\hline & $-\mathbf{A L A}$ & + ALA & $-\mathbf{A L A}$ & + ALA \\
\hline Chlorophyll a $\left(\mathrm{mg} \cdot \mathrm{g}^{-1} \mathrm{FW}\right)$ & $2.44 \pm 0.11^{b}$ & $2.86 \pm 0.13^{a}$ & $1.78 \pm 0.16^{\mathrm{d}}$ & $2.11 \pm 0.12^{c}$ \\
\hline Chlorophyll b $\left(\mathrm{mg} \cdot \mathrm{g}^{-1} \mathrm{FW}\right)$ & $1.21 \pm 0.08^{b}$ & $1.68 \pm 0.07^{\mathrm{a}}$ & $0.92 \pm 0.05^{\mathrm{d}}$ & $1.18 \pm 0.08^{\mathrm{c}}$ \\
\hline Total Chl $\left(\mathrm{mg} \cdot \mathrm{g}^{-1} \mathrm{FW}\right)$ & $3.65 \pm 0.13^{b}$ & $4.54 \pm 0.11^{\mathrm{a}}$ & $2.70 \pm 0.14^{\mathrm{d}}$ & $3.29 \pm 0.10^{c}$ \\
\hline Carotenoid $\left(\mathrm{mg} \cdot \mathrm{g}^{-1} \mathrm{FW}\right)$ & $0.29 \pm 0.04^{b}$ & $0.34 \pm 0.08^{a}$ & $0.22 \pm 0.03^{c}$ & $0.28 \pm 0.04^{b}$ \\
\hline Proteins $\left(\mathrm{mg} \cdot \mathrm{g}^{-1} \mathrm{FW}\right)$ & $0.89 \pm 0.08^{b}$ & $1.03 \pm 0.09^{\mathrm{a}}$ & $0.78 \pm 0.11^{\mathrm{d}}$ & $0.85 \pm 0.12^{c}$ \\
\hline Soluble sugars $\left(\mu \mathrm{g} \cdot \mathrm{g}^{-1} \mathrm{FW}\right)$ & $2.04 \pm 0.13^{b}$ & $2.19 \pm 0.14^{\mathrm{a}}$ & $1.87 \pm 0.12^{\mathrm{d}}$ & $1.96 \pm 0.14^{\mathrm{c}}$ \\
\hline Proline $\left(\mu \mathrm{g} \cdot \mathrm{g}^{-1} \mathrm{FW}\right)$ & $14.8 \pm 0.38^{\mathrm{d}}$ & $22.7 \pm 0.44^{\mathrm{c}}$ & $26.7 \pm 0.38^{b}$ & $32.6 \pm 0.46^{\mathrm{a}}$ \\
\hline Total Phenolics $\left(\mu \mathrm{mol} \cdot \mathrm{g}^{-1} \mathrm{FW}\right)$ & $13.9 \pm 0.36^{b}$ & $15.2 \pm 0.32^{\mathrm{a}}$ & $09.1 \pm 0.29^{d}$ & $11.8 \pm 0.31^{\mathrm{c}}$ \\
\hline Total Flavonoids $\left(\mu \mathrm{mol} \cdot \mathrm{g}^{-1} \mathrm{FW}\right)$ & $6.23 \pm 0.13^{b}$ & $8.25 \pm 0.16^{\mathrm{a}}$ & $4.14 \pm 0.14^{\mathrm{d}}$ & $5.07 \pm 0.16^{c}$ \\
\hline $\mathrm{H}_{2} \mathrm{O}_{2}\left(\mu \mathrm{mol} \cdot \mathrm{g}^{-1} \mathrm{FW}\right)$ & $0.25 \pm 0.03^{c}$ & $0.21 \pm 0.06^{\mathrm{d}}$ & $0.68 \pm 0.05^{\mathrm{a}}$ & $0.51 \pm 0.04^{b}$ \\
\hline MDA $\left(\mu \mathrm{mol} \cdot \mathrm{g}^{-1} \mathrm{FW}\right)$ & $0.19 \pm 0.04^{\mathrm{c}}$ & $0.17 \pm 0.03^{\mathrm{d}}$ & $0.51 \pm 0.05^{\mathrm{a}}$ & $0.32 \pm 0.04^{b}$ \\
\hline $\mathrm{DPPH}\left(\mathrm{IC}_{50}, \mu \mathrm{g} \cdot \mathrm{mL}^{-1}\right)$ & $0.36 \pm 0.04^{\mathrm{a}}$ & $0.34 \pm 0.03^{b}$ & $0.33 \pm 0.04^{\mathrm{c}}$ & $0.32 \pm 0.02^{\mathrm{d}}$ \\
\hline
\end{tabular}

Different alphabetical letters in the same row exhibit significant differences among the applied treatments $(p \leq 0.05)$. 
Table 8. Contents of chlorophyll, carotenoids, proteins, sugars, proline, phenolics, flavonoids, $\mathrm{H}_{2} \mathrm{O}_{2}$, MDA and antioxidant activity (DPPH) in leaves of Bigo cultivar under salt and/or ALA (7 ppm) treatments.

\begin{tabular}{|c|c|c|c|c|}
\hline \multirow{2}{*}{$\mathrm{NaCl}$ Treatment } & \multicolumn{2}{|c|}{$0 \mathrm{mM}$} & \multicolumn{2}{|c|}{$100 \mathrm{mM}$} \\
\hline & $-\mathbf{A L A}$ & + ALA & - ALA & + ALA \\
\hline Chlorophyll a $\left(\mathrm{mg} \cdot \mathrm{g}^{-1} \mathrm{FW}\right)$ & $2.47 \pm 0.12^{b}$ & $2.68 \pm 0.17^{a}$ & $1.81 \pm 0.14^{\mathrm{d}}$ & $2.23 \pm 0.15^{c}$ \\
\hline Chlorophyll b $\left(\mathrm{mg} \cdot \mathrm{g}^{-1} \mathrm{FW}\right)$ & $1.18 \pm 0.04^{b}$ & $1.44 \pm 0.07^{\mathrm{a}}$ & $0.88 \pm 0.06^{\mathrm{c}}$ & $1.19 \pm 0.06^{b}$ \\
\hline Total Chl $\left(\mathrm{mg} \cdot \mathrm{g}^{-1} \mathrm{FW}\right)$ & $3.65 \pm 0.15^{b}$ & $4.12 \pm 0.12^{\mathrm{a}}$ & $2.69 \pm 0.11^{\mathrm{d}}$ & $3.42 \pm 0.12^{c}$ \\
\hline Carotenoid $\left(\mathrm{mg} \cdot \mathrm{g}^{-1} \mathrm{FW}\right)$ & $0.27 \pm 0.05^{b}$ & $0.32 \pm 0.04^{\mathrm{a}}$ & $0.18 \pm 0.06^{\mathrm{d}}$ & $0.24 \pm 0.05^{c}$ \\
\hline Proteins $\left(\mathrm{mg} \cdot \mathrm{g}^{-1} \mathrm{FW}\right)$ & $0.82 \pm 0.07^{b}$ & $1.08 \pm 0.09^{\mathrm{a}}$ & $0.61 \pm 0.12^{\mathrm{c}}$ & $0.83 \pm 0.11^{b}$ \\
\hline Soluble sugars $\left(\mu \mathrm{g} \cdot \mathrm{g}^{-1} \mathrm{FW}\right)$ & $2.01 \pm 0.11^{b}$ & $2.23 \pm 0.11^{\mathrm{a}}$ & $1.81 \pm 0.13^{\mathrm{d}}$ & $1.93 \pm 0.11^{\mathrm{c}}$ \\
\hline Proline $\left(\mu \mathrm{g} \cdot \mathrm{g}^{-1} \mathrm{FW}\right)$ & $14.1 \pm 0.31^{d}$ & $21.2 \pm 0.37^{c}$ & $27.5 \pm 0.41^{b}$ & $31.3 \pm 0.27^{\mathrm{a}}$ \\
\hline Total Phenolics $\left(\mu \mathrm{mol} \cdot \mathrm{g}^{-1} \mathrm{FW}\right)$ & $12.8 \pm 0.41^{b}$ & $15.7 \pm 0.27^{\mathrm{a}}$ & $08.3 \pm 0.32^{d}$ & $12.1 \pm 0.33^{c}$ \\
\hline Total Flavonoids $\left(\mu \mathrm{mol} \cdot \mathrm{g}^{-1} \mathrm{FW}\right)$ & $6.11 \pm 0.11^{b}$ & $8.12 \pm 0.12^{a}$ & $4.03 \pm 0.16^{\mathrm{d}}$ & $5.22 \pm 0.15^{c}$ \\
\hline $\mathrm{H}_{2} \mathrm{O}_{2}\left(\mu \mathrm{mol} \cdot \mathrm{g}^{-1} \mathrm{FW}\right)$ & $0.23 \pm 0.05^{c}$ & $0.20 \pm 0.05^{d}$ & $0.71 \pm 0.06^{\mathrm{a}}$ & $0.57 \pm 0.07^{b}$ \\
\hline MDA $\left(\mu \mathrm{mol} \cdot \mathrm{g}^{-1} \mathrm{FW}\right)$ & $0.18 \pm 0.06^{c}$ & $0.15 \pm 0.06^{\mathrm{d}}$ & $0.59 \pm 0.08^{a}$ & $0.39 \pm 0.06^{b}$ \\
\hline $\mathrm{DPPH}\left(\mathrm{IC}_{50}, \mu \mathrm{g} \cdot \mathrm{mL}^{-1}\right)$ & $0.34 \pm 0.03^{a}$ & $0.32 \pm 0.04^{b}$ & $0.31 \pm 0.03^{c}$ & $0.29 \pm 0.03^{d}$ \\
\hline
\end{tabular}

Different alphabetical letters in the same row show significant differences among the applied treatments $(p \leq 0.05)$.

Table 9. Contents of chlorophyll, carotenoids, proteins, sugars, proline, phenolics, flavonoids, $\mathrm{H}_{2} \mathrm{O}_{2}$, MDA and antioxidant activity (DPPH) in leaves of Cambrinus cultivar under salt and/or ALA (7 ppm) treatments.

\begin{tabular}{|c|c|c|c|c|}
\hline \multirow{2}{*}{$\mathrm{NaCl}$ Treatment } & \multicolumn{2}{|c|}{$0 \mathrm{mM}$} & \multicolumn{2}{|c|}{$100 \mathrm{mM}$} \\
\hline & -ALA & + ALA & $-\mathbf{A L A}$ & + ALA \\
\hline Chlorophyll a (mg.g $\left.{ }^{-1} \mathrm{FW}\right)$ & $2.39 \pm 0.12^{b}$ & $2.72 \pm 0.15^{\mathrm{a}}$ & $1.71 \pm 0.13^{\mathrm{d}}$ & $2.22 \pm 0.11^{\mathrm{c}}$ \\
\hline Chlorophyll b $\left(\mathrm{mg} \cdot \mathrm{g}^{-1} \mathrm{FW}\right)$ & $1.28 \pm 0.05^{b}$ & $1.56 \pm 0.08^{\mathrm{a}}$ & $0.91 \pm 0.04^{d}$ & $1.21 \pm 0.07^{\mathrm{c}}$ \\
\hline Total Chl $\left(\mathrm{mg} \cdot \mathrm{g}^{-1} \mathrm{FW}\right)$ & $3.67 \pm 0.12^{b}$ & $4.28 \pm 0.11^{\mathrm{a}}$ & $2.62 \pm 0.13^{d}$ & $3.43 \pm 0.12^{c}$ \\
\hline Carotenoid $\left(\mathrm{mg} \cdot \mathrm{g}^{-1} \mathrm{FW}\right)$ & $0.27 \pm 0.05^{b}$ & $0.36 \pm 0.06^{\mathrm{d}}$ & $0.20 \pm 0.04^{c}$ & $0.25 \pm 0.05^{c}$ \\
\hline Proteins $\left(\mathrm{mg} \cdot \mathrm{g}^{-1} \mathrm{FW}\right)$ & $0.86 \pm 0.07^{b}$ & $1.15 \pm 0.07^{\mathrm{a}}$ & $0.71 \pm 0.12^{\mathrm{d}}$ & $0.82 \pm 0.13^{c}$ \\
\hline Soluble sugars $\left(\mu \mathrm{g} \cdot \mathrm{g}^{-1} \mathrm{FW}\right)$ & $1.98 \pm 0.11^{\mathrm{c}}$ & $2.21 \pm 0.12^{\mathrm{a}}$ & $1.81 \pm 0.11^{\mathrm{d}}$ & $2.06 \pm 0.12^{b}$ \\
\hline Proline $\left(\mu \mathrm{g} \cdot \mathrm{g}^{-1} \mathrm{FW}\right)$ & $16.1 \pm 0.31^{d}$ & $24.2 \pm 0.33^{c}$ & $27.2 \pm 0.34^{b}$ & $33.4 \pm 0.38^{a}$ \\
\hline Total Phenolics $\left(\mu \mathrm{mol} \cdot \mathrm{g}^{-1} \mathrm{FW}\right)$ & $14.1 \pm 0.34^{b}$ & $15.6 \pm 0.35^{\mathrm{a}}$ & $10.2 \pm 0.37^{\mathrm{d}}$ & $13.7 \pm 0.33^{c}$ \\
\hline Total Flavonoids $\left(\mu \mathrm{mol} \cdot \mathrm{g}^{-1} \mathrm{FW}\right)$ & $6.88 \pm 0.12^{b}$ & $8.11 \pm 0.14^{\mathrm{a}}$ & $4.03 \pm 0.12^{\mathrm{d}}$ & $5.04 \pm 0.13^{c}$ \\
\hline $\mathrm{H}_{2} \mathrm{O}_{2}\left(\mu \mathrm{mol} \cdot \mathrm{g}^{-1} \mathrm{FW}\right)$ & $0.23 \pm 0.07^{\mathrm{c}}$ & $0.20 \pm 0.05^{\mathrm{d}}$ & $0.72 \pm 0.07^{\mathrm{a}}$ & $0.55 \pm 0.06^{b}$ \\
\hline $\operatorname{MDA}\left(\mu \mathrm{mol} \cdot \mathrm{g}^{-1} \mathrm{FW}\right)$ & $0.18 \pm 0.05^{\mathrm{c}}$ & $0.15 \pm 0.04^{\mathrm{d}}$ & $0.56 \pm 0.04^{\mathrm{a}}$ & $0.38 \pm 0.03^{b}$ \\
\hline $\mathrm{DPPH}\left(\mathrm{IC}_{50}, \mu \mathrm{g} \cdot \mathrm{mL}^{-1}\right)$ & $0.35 \pm 0.02^{\mathrm{a}}$ & $0.33 \pm 0.03^{b}$ & $0.32 \pm 0.02^{\mathrm{c}}$ & $0.31 \pm 0.02^{\mathrm{d}}$ \\
\hline
\end{tabular}

Different alphabetical letters in the same row show significant differences among the applied treatments $(p \leq 0.05)$.

Figure 3 indicates that antioxidant enzyme (APX, POD and CAT) activities in Slaski 2 leaves were significantly activated under salinity stress. Additionally, treating Slaski 2 genotype with ALA under salt stress significantly augmented enzyme activities, compared to plants dealt only with salt (Figure 3). Furthermore, ALA significantly promoted antioxidant enzyme activities in non-stressed Slaski 2 plants, relative to control plants. On the other hand, Figure 4 indicates that Carmen leaves subjected to $\mathrm{NaCl}$ stress exhibited non-significantly induced levels of antioxidant enzyme activities, as compared to control samples. Such an increase was less than that reported in Slaski 2 variety. ALA significantly augmented the activities of antioxidant enzymes in Carmen genotype subjected to salt stress, relative to $\mathrm{NaCl}$-stressed plants (Figure 4). Furthermore, ALA significantly augmented antioxidant enzyme activities in non-stressed Carmen plants, as compared to control plants. 


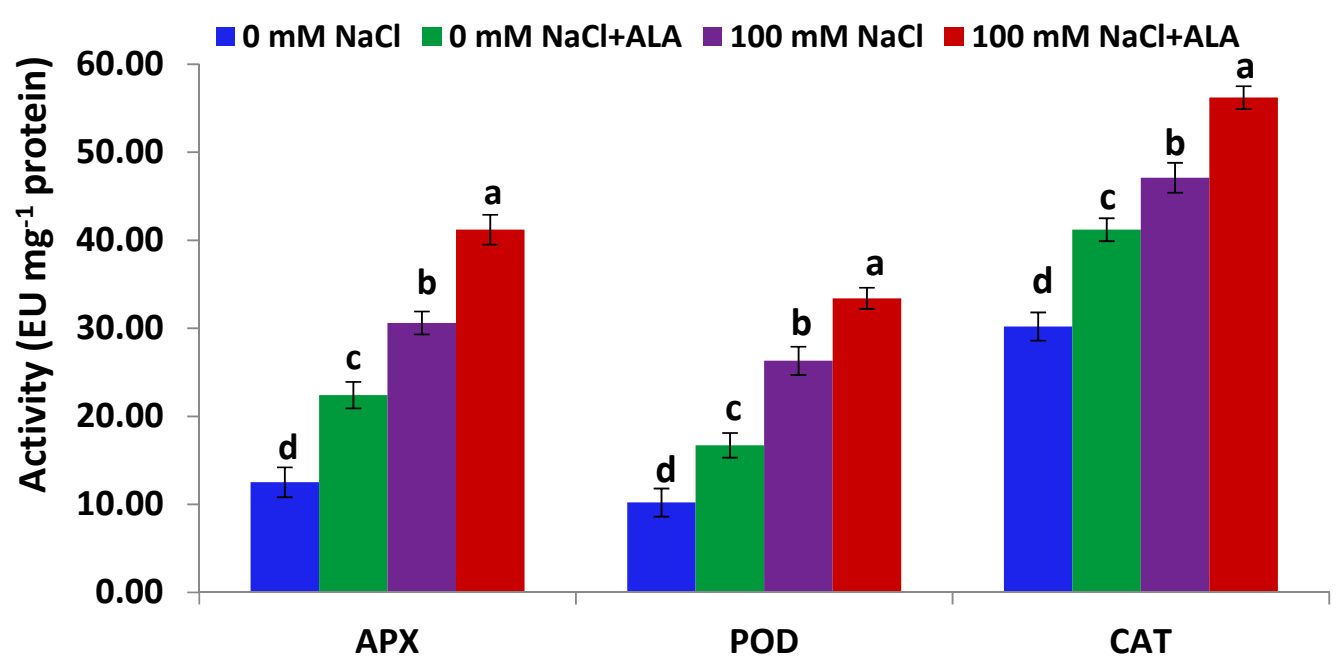

Figure 3. APX, POD and CAT activities in Slaski 2 cultivar leaves under salt and/or ALA (7 ppm) effects. Values represent means $\pm \mathrm{SE}(n=4)$. Different letters show significant difference among treatments $(p \leq 0.05)$.

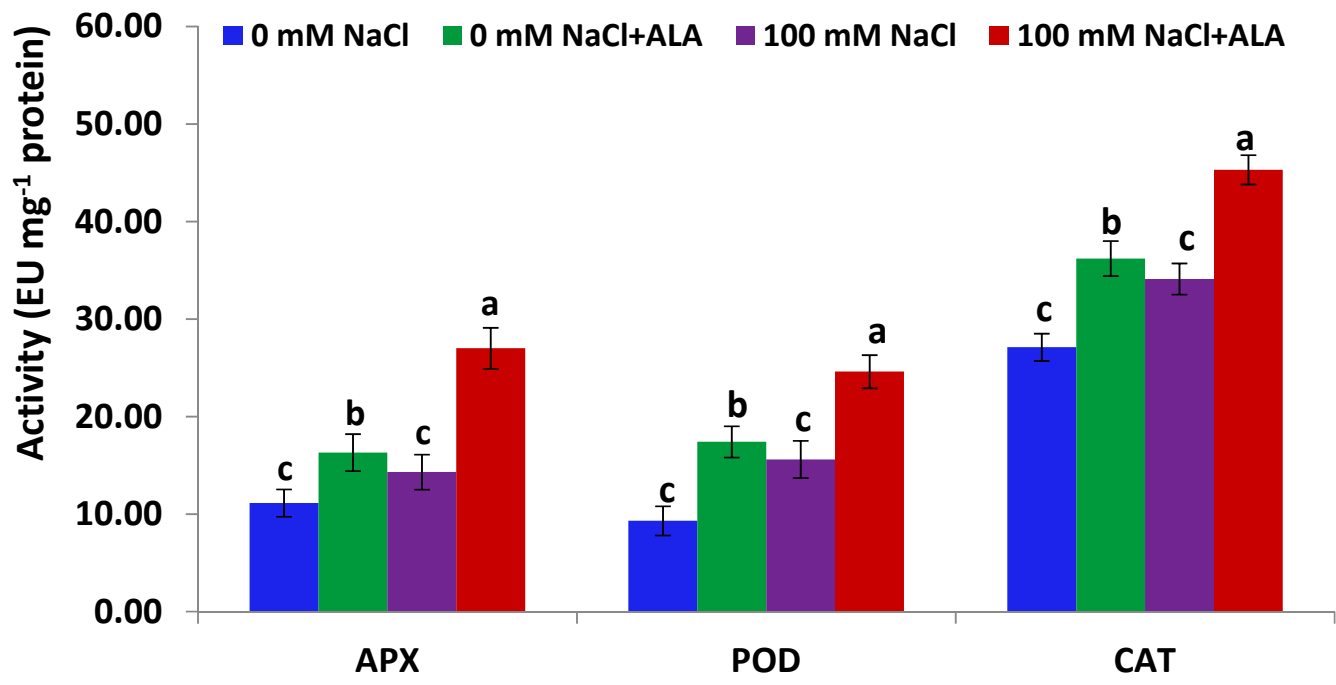

Figure 4. APX, POD and CAT activities in Carmen cultivar leaves under salt and/or ALA (7 ppm) effects. Values represent means $\pm \mathrm{SE}(n=4)$. Different letters show significant difference among treatments $(p \leq 0.05)$.

Figure 5 indicates antioxidant genes ( $A P X, C A T$ and $S O D)$ expression in Slaski 2 leaves was significantly up-regulated under high salinity conditions, as compared to control plants. ALA significantly enhanced antioxidant genes expression in Slaski 2 plants subjected to $\mathrm{NaCl}$ stress, relative to $\mathrm{NaCl}$-treated plants (Figure 5). Moreover, ALA treatment significantly augmented antioxidant genes expression in non-stressed Slaski 2, relative to control plants. Figure 6 indicates that antioxidant genes expression in Carmen leaves was non-significantly enhanced under $\mathrm{NaCl}$ conditions, relative to control plants. Such an increase was less than that reported in Slaski 2 leaves. ALA significantly augmented the antioxidant genes expression in the salt-stressed Carmen plants, as compared to $\mathrm{NaCl}$-treated plants (Figure 6). Moreover, ALA significantly enhanced the antioxidant genes expression in the non-stressed Carmen plants, as compared to control plants. 


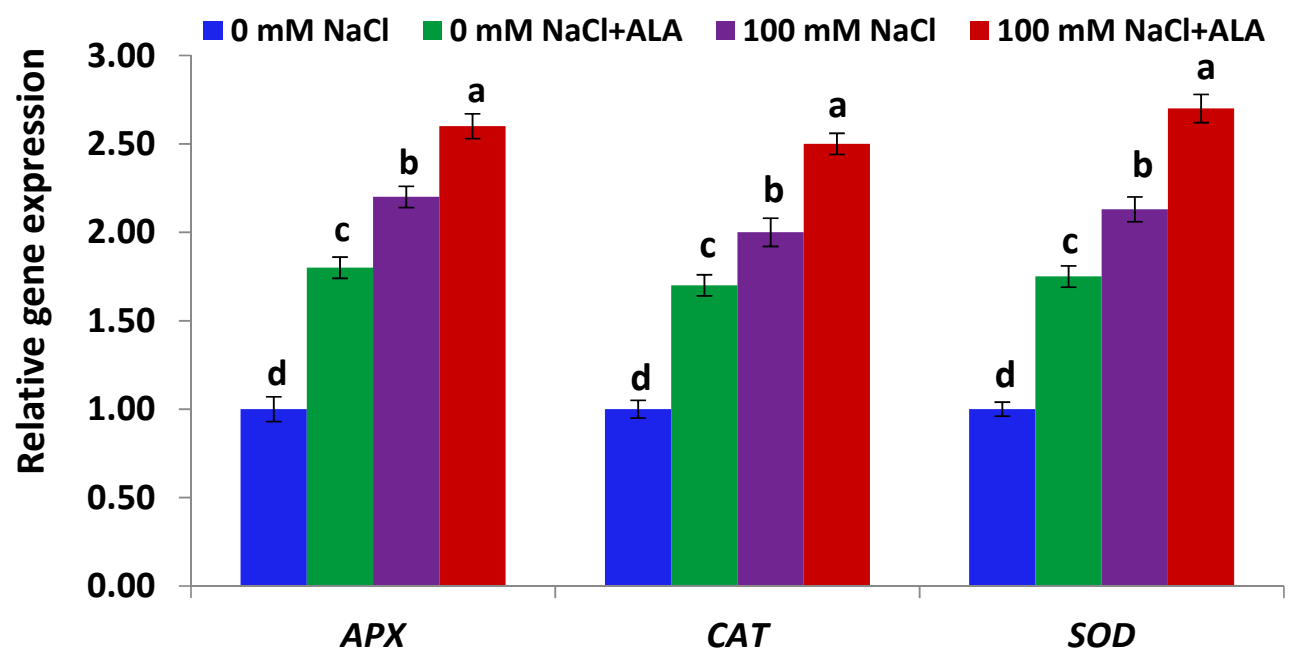

Figure 5. Expression level of antioxidant genes in Slaski 2 cultivar leaves under salt and/or ALA $(7 \mathrm{ppm})$ effects. Values represent means $\pm \mathrm{SE}(n=4)$. Different letters show significant difference among treatments $(p \leq 0.05)$.

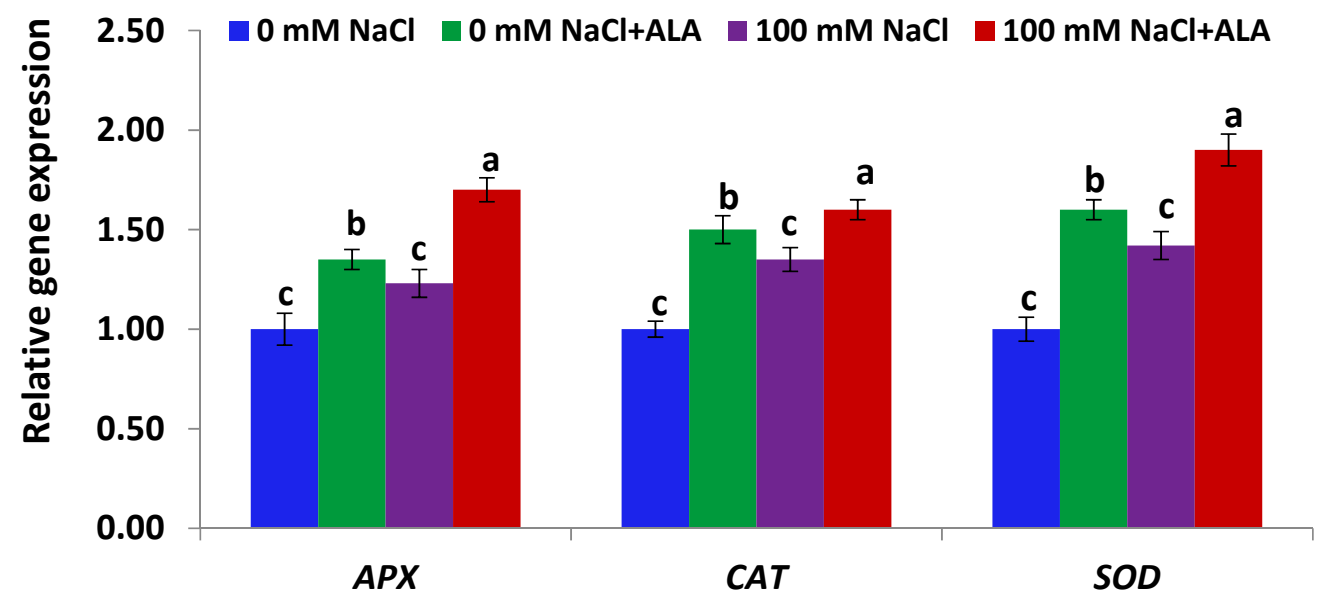

Figure 6. Expression level of antioxidant genes in Carmen cultivar leaves under salt and/or ALA $(7 \mathrm{ppm})$ effects. Values represent means $\pm \mathrm{SE}(n=4)$. Different letters show significant difference among treatments $(p \leq 0.05)$.

The above results showed Slaski 2, Damseaux and Urbanowicki genotypes tolerated salt stress more than Carmen, Bigo and Cambrinus genotypes. This result suggests that Slaski 2, Damseaux and Urbanowicki genotypes (having the highest diversity level) are moderately tolerant to salt stress, whereas Carmen, Bigo and Cambrinus genotypes (having the lowest diversity level) are sensitive to salt stress, suggesting sort of correlation between level of variability and degree of salinity tolerance. Moreover, results showed that Slaski 2, Damseaux and Urbanowicki plants enhanced their self-defense mechanisms by up-regulating their antioxidant enzymes under high salinity stress to alleviate the resulting oxidative damage. Results also showed a key role of ALA to further improve salinity tolerance in those six barley cultivars by up-regulating photosynthetic pigment biosynthetic pathway and expression of antioxidant genes. ALA has played key roles against the abiotic stresses-induced inhibitory in various plants. ALA Foliar spray alleviated the adverse effects of high salinity in creeping bentgrass [47]. Exogenous ALA also augmented salinity tolerance in rice [48], tomato [49], sicklepod [50], swiss chard [51], and cucumber [52]. Additionally, exogenous ALA boosted chlorophyll content in lettuce subjected to UV-B stress [53]. ALA also increased chlorophyll fluorescence indices in rapes exposed to drought conditions [54]. Furthermore, ALA also induced expression of genes mediating photosynthesis Calvin cycle in rapes exposed to drought stress [55]. 
In conclusion, the current study reported a considerable AFLP-based genetic diversity level in the barley germplasm studied and might be exploited to improve crop yield and quality. Results also indicated a correlation between level of variability and degree of salinity tolerance in barley cultivars. ALA improved salt tolerance in barley cultivars.

\section{Materials and Methods}

\subsection{Plant Material}

Forty European barley accessions were brought from the Center for Genetic Resources in Netherlands (Table 2). Those varieties included 16 genotypes originated from France, 12 genotypes from the Netherlands, and 12 genotypes from Poland.

\subsection{Molecular Diversity Analysis}

\subsubsection{DNA Isolation and AFLP Analysis}

Total DNA was prepared from the 4-week old leaf tissues of each genotype by DNeasy Plant Mini Kit (Qiagen, Hilden, Germany). Five DNA samples were isolated from each of the 40 genotypes and used for AFLP analyses. AFLP analyses were done as reported by Vos et al. [56] with little changes as shown in Adawy et al. [44]. Briefly, DNA samples were digested with MseI and EcoRI for $2 \mathrm{~h}$ at $37^{\circ} \mathrm{C}$, then left at $70{ }^{\circ} \mathrm{C}$ for $15 \mathrm{~min}$., then ligated to MseI and EcoRI adapters for $4 \mathrm{~h}$ at $20^{\circ} \mathrm{C}$. Preamplification was conducted in a DNA thermocycler (Applied Biosystems, Foster City, CA, USA) for 20 cycles $\left(94{ }^{\circ} \mathrm{C} / 30 \mathrm{~s}, 56^{\circ} \mathrm{C} / 60 \mathrm{~s}\right.$ and $\left.72{ }^{\circ} \mathrm{C} / 60 \mathrm{~s}\right)$. Amplified products were then diluted and used for selective amplification. Selective amplification reactions were done using 14 primer pairs (Eurogentec SA) reported by Adawy et al. [44] in a DNA thermocycler set up as follows: one cycle at $94{ }^{\circ} \mathrm{C} / 30 \mathrm{~s}$, $65^{\circ} \mathrm{C} / 30 \mathrm{~s}$ and $72{ }^{\circ} \mathrm{C} / 60 \mathrm{~s}$, followed by 13 cycles during which the annealing temperature decreased by $0.7{ }^{\circ} \mathrm{C}$ every cycle. This was then followed by 23 cycles at $94{ }^{\circ} \mathrm{C} / 30 \mathrm{~s}, 56{ }^{\circ} \mathrm{C} / 30 \mathrm{~s}$ and $72{ }^{\circ} \mathrm{C} / 60 \mathrm{~s}$. The 14 primer sets comprised E-AAC/M-CTT, E-ACT/M-CTG, E-ACA/M-CAG, E-ACT/M-CAG, E-AGG/M-CTA, E-AAG/M-CTT, E-ACT/M-CTT, E-ACC/M-CTA, E-AGG/M-CAG, E-AAG/M-CTG, E-AGG/M-CAC, E-ACC/M-CAC, E-AGG/M-CTT, and E-AGG/M-CAG (Table 1). Polyacrylamide gels $(8 \%, w / v)$ were used to analyze amplified PCR products. SYBR Gold stain was used to stain gels.

\subsubsection{Data Analysis}

AFLP fragments were represented as present (1) and absent (0), forming binary data sets. PowerMarker 3.25 [57] and GenAlEx 6.5 [58] were used to estimate variability indices such as polymorphism, observed heterozygosity $\left(H_{0}\right)$, fixation index $(F)$, polymorphic information content (PIC), gene diversity, and expected heterozygosity $\left(H_{e}\right)$. UPGMA cluster analysis was carried out based on Nei's genetic distance [59].

The genetic structure of the 40 barley genotypes was studied using STRUCTURE 2.3 software [60]. The analyses were done using hypothetical populations numbers $(K)$ ranging from 1 to 11 , along with 100,000 burn-in run iteration as well as 100,000 Markov chain Monte Carlo, and with ten runs for each $K$ value. Structure Harvester [61] was applied and showed the best likely values of $K$ [62].

\subsection{Salt Tolerance Test and 5-Aminolevulinic Acid Effects on Barley}

\subsubsection{Growth Condition and Treatments}

Barley cultivars (Slaski 2, Damseaux, Urbanowicki, Carmen, Bigo and Cambrinus) seeds were sterilized for $4 \mathrm{~min}$ in $5 \% \mathrm{NaClO}$ solution, washed five times in distilled $\mathrm{H}_{2} \mathrm{O}$, and left to grow in a growth chamber for 5 days. Barley seedlings were then transferred into plastic pots (one seedling per pot, five replicates) having soil comprising perlite, sand, and peat (1:1:1). Randomized pots were kept under conditions of $16 / 8 \mathrm{~h}, 26 / 21^{\circ} \mathrm{C}$, and $80 \%$ humidity. After transplantation immediately, 
plants were watered daily using Hoagland nutrient containing 0 and $100 \mathrm{mM} \mathrm{NaCl}$ over 3 weeks. 5-aminolevulinic acid foliar sprays ( $7 \mathrm{ppm}$ ) were applied weekly (i.e., every 7 days) to barley plants. After 3 weeks of transplantation, leaves were harvested for physiological analyses.

\subsubsection{Estimation of Contents of Photosynthetic Pigments}

Leaf chlorophyll and carotenoid contents were measured spectrophotometrically as indicated by Lichtenthaler and Wellburn [63]. Absorbance was spectrophotometrically measured at 452.5, 663, 644, and $452.5 \mathrm{~nm}$ for carotenoids, chl a and chl b, respectively. $80 \%$ acetone was served as a blank.

\subsubsection{Estimation of Osmolytes, $\mathrm{H}_{2} \mathrm{O}_{2}, \mathrm{MDA}$, and Antioxidant Activity (DPPH)}

Total leaf protein content was calculated using Bradford protocol [64]. Total soluble sugar was determined as indicated by Dey [65], and absorbance was reported at $485 \mathrm{~nm}$. Leaf proline content was calculated as mentioned by Bates et al. [66].

Hydrogen peroxide $\left(\mathrm{H}_{2} \mathrm{O}_{2}\right)$ was measured as reported by Velikova et al. [67]. Malondialdehyde (MDA) was calculated as reported by Heath and Packer [68].

Total leaf phenolic content was determined as reported by Zieslin and Ben-Zaken [69]. Total leaf flavonoid content was investigated following Zhishen et al. [70]. Optical density was taken at $510 \mathrm{~nm}$. Leaf antioxidant capacity was estimated using 2,2'-diphenypicrylhydrazyl (DPPH) [71]. Absorbance was taken at $517 \mathrm{~nm}$.

\subsubsection{Antioxidant Enzyme Assay}

Peroxidase (POD), ascorbate peroxidase (APX) and catalase (CAT) activities were investigated in fresh leaves using the protocol of Zhang and Kirkham [72]. Optical density was measured at $290 \mathrm{~nm}$ (APX) or $470 \mathrm{~m}$ (POD) or $240 \mathrm{~nm}$ (CAT).

\subsubsection{Transcription Analyses}

Quantitative real-time PCR (qRT-PCR) analyses were conducted to evaluate expressions of 3 genes conferring salt tolerance in barley leaves. These three genes represent antioxidants genes (APX, CAT and $S O D$ ). Total RNA extraction was carried out from barley leaves by RNeasy Plant Mini kit (Qiagen). cDNA synthesis was made by Qiagen Reverse Transcription kit. Quantitative RT-PCR was done by Qiagen QuantiTect SYBR Green PCR kit. The conditions of PCR amplifications were: $10 \mathrm{~min}$ at $95^{\circ} \mathrm{C}$; 40 cycles of $20 \mathrm{~s}$ at $94{ }^{\circ} \mathrm{C}, 30 \mathrm{~s}$ at $60^{\circ} \mathrm{C}, 2 \mathrm{~min}$ at $72{ }^{\circ} \mathrm{C}$; and $4 \mathrm{~min}$ at $72{ }^{\circ} \mathrm{C}$. Gene specific-primers were used for PCR amplification [17]. Melting-curve analyses were then applied. UBIQUITIN (UBQ1) was used for normalization, and the relative expression was determined by $2^{-\Delta \Delta C t}$ method.

\subsubsection{Statistical Analysis}

One-way analysis of variance (ANOVA) as well as Duncan's multiple range tests were performed. Values at $p \leq 0.05$ differ significantly. Data represent means \pm SE ( $n=4$ representing different plants).

Author Contributions: M.A.E. conducted all the experiments and data analyses and wrote, revised, and approved the manuscript draft. I.A.A., A.A.A. (Abdulaziz A. Alsahli), H.M.A. and A.A.A. (Aisha A. Alayafi) helped in data analysis and approved the manuscript draft. J.W. and M.A. revised and approved the manuscript draft.

Funding: This research was funded by Deanship of Scientific Research at King Saud University No. RG-1438-036. Acknowledgments: Authors would like to thank Tanta University and its active Magdy Sabaa for supporting this work.

Conflicts of Interest: The authors declare no conflict of interest.

\section{References}

1. Feuillet, C.; Langridge, P.; Waugh, R. Cereal breeding takes a walk on the wild side. Trends Genet. 2007, 24, 25-32. [CrossRef] [PubMed] 
2. Ferreira, J.R.; Pereira, J.F.; Turchetto, C.; Minella, E.; Consoli, L.; Delatorre, C.A. Assessment of genetic diversity in Brazilian barley using SSR markers. Genet. Mol. Biol. 2016, 39, 86-96. [CrossRef] [PubMed]

3. Goyal, A.; Ahmed, M. Barley: Production, improvement, and uses. Crop Sci. 2012, 52, 2852-2854.

4. Minella, E. Melhoramento de cevada. In Melhoramento de Espécies Cultivadas, 2nd ed.; Borém, A., Ed.; Editora UFV: Viçosa, Brazil, 2005; pp. 275-299.

5. Ledovskoy, Y.; Abugalieva, S.; Turuspekov, Y. Comparative assessment of the genetic variation in wild and cultivated barley based on SSR markers. Asian Australas J. Plant Sci. Biotechnol. 2010, 4, 21-26.

6. Nandha, P.S.; Singh, J. Comparative assessment of genetic diversity between wild and cultivated barley using Gssr and EST-SSR markers. Plant Breed. 2014, 133, 28-35. [CrossRef]

7. Nevo, E.; Fu, Y.B.; Pavlicek, T.; Khalifa, S.; Tavasi, M.; Beiles, A. Evolution of wild cereals during 28 years of global warming in Israel. Proc. Natl. Acad. Sci. USA 2012, 109, 3412-3415. [CrossRef] [PubMed]

8. El-Esawi, M.A.; Sammour, R. Karyological and phylogenetic studies in the genus Lactuca L. (asteraceae). Cytologia 2014, 79, 269-275. [CrossRef]

9. El-Esawi, M.A. Genetic diversity and evolution of Brassica genetic resources: From morphology to novel genomic technologies-A review. Plant Genet. Resour. 2017, 15, 388-399. [CrossRef]

10. El-Esawi, M.A.; Germaine, K.; Bourke, P.; Malone, R. Genetic diversity and population structure of Brassica oleracea germplasm in Ireland using SSR markers. C. R. Biol. 2016, 339, 133-140. [CrossRef] [PubMed]

11. El-Esawi, M.A.; Germaine, K.; Bourke, P.; Malone, R. AFLP analysis of genetic diversity and phylogenetic relationships of Brassica oleracea in Ireland. C. R. Biol. 2016, 339, 163-170. [CrossRef] [PubMed]

12. El-Esawi, M.A.; Mustafa, A.; Badr, S.; Sammour, R. Isozyme analysis of genetic variability and population structure of Lactuca L. germplasm. Biochem. Syst. Ecol. 2017, 70, 73-79. [CrossRef]

13. El-Esawi, M.A. SSR analysis of genetic diversity and structure of the germplasm of faba bean (Vicia faba L.). C. R. Biol. 2017, 340, 474-480. [CrossRef] [PubMed]

14. El-Esawi, M.A.; Witczak, J.; Abomohra, A.; Ali, H.M.; Elshikh, M.S.; Ahmad, M. Analysis of the Genetic Diversity and Population Structure of Austrian and Belgian Wheat Germplasm Within a Regional Context Based on DArT Markers. Genes 2018, 9, 47. [CrossRef] [PubMed]

15. El-Esawi, M.A.; Elkelish, A.; Elansary, H.O.; Ali, H.M.; Elshikh, M.; Witczak, I.; Ahmad, M. Genetic Transformation and Hairy Root Induction Enhance the Antioxidant Potential of Lactuca serriola L. Oxid. Med. Cell. Long. 2017, 2017, 5604746. [CrossRef] [PubMed]

16. El-Esawi, M.A.; Elansary, H.O.; El-Shanhorey, N.A.; Abdel-Hamid, A.M.E.; Ali, H.M.; Elshikh, M.S. Salicylic Acid-Regulated Antioxidant Mechanisms and Gene Expression Enhance Rosemary Performance under Saline Conditions. Front. Physiol. 2017, 8, 716. [CrossRef] [PubMed]

17. Vwioko, E.; Adinkwu, O.; El-Esawi, M.A. Comparative Physiological, Biochemical and Genetic Responses to Prolonged Waterlogging Stress in Okra and Maize Given Exogenous Ethylene Priming. Front. Physiol. 2017, 8, 632. [CrossRef] [PubMed]

18. Consentino, L.; Lambert, S.; Martino, C.; Jourdan, N.; Bouchet, P.-E.; Witczak, J.; Castello, P.; El-Esawi, M.; Corbineau, F.; d'Harlingue, A.; et al. Blue-light dependent reactive oxygen species formation by Arabidopsis cryptochrome may defi ne a novel evolutionarily conserved signalling mechanism. New Phytol. 2015, 206, 1450-1462. [CrossRef] [PubMed]

19. Elansary, H.O.; Szopa, A.; Kubica, P.; Ekiert, H.; Ali, H.M.; Elshikh, M.S.; Abdel-Salam, E.M.; El-Esawi, M.; El-Ansary, D.O. Bioactivities of traditional medicinal plants in Alexandria. Evid. Based Complement. Altern. Med. 2018, 2018, 1463579. [CrossRef] [PubMed]

20. Elansary, H.O.; Yessoufou, K.; Abdel-Hamid, A.M.E.; El-Esawi, M.A.; Ali, H.M.; Elshikh, M.S. Seaweed Extracts Enhance Salam Turfgrass Performance during Prolonged Irrigation Intervals and Saline Shock. Front. Plant. Sci. 2017, 8, 830. [CrossRef] [PubMed]

21. El-Esawi, M.A. Micropropagation technology and its applications for crop improvement. In Plant Tissue Culture: Propagation, Conservation and Crop Improvement; Anis, M., Ahmad, N., Eds.; Springer: Singapore, 2016; pp. 523-545.

22. El-Esawi, M.A. Nonzygotic embryogenesis for plant development. In Plant Tissue Culture: Propagation, Conservation and Crop Improvement; Anis, M., Ahmad, N., Eds.; Springer: Singapore, 2016; pp. 583-598.

23. El-Esawi, M.A. Somatic hybridization and microspore culture in Brassica improvement. In Plant Tissue Culture: Propagation, Conservation and Crop Improvement; Anis, M., Ahmad, N., Eds.; Springer: Singapore, 2016; pp. 599-609. 
24. El-Esawi, M.; Arthaut, L.; Jourdan, N.; d’Harlingue, A.; Martino, C.; Ahmad, M. Blue-light induced biosynthesis of ROS contributes to the signaling mechanism of Arabidopsis cryptochrome. Sci. Rep. 2017, 7, 13875. [CrossRef] [PubMed]

25. El-Esawi, M.; Glascoe, A.; Engle, D.; Ritz, T.; Link, J.; Ahmad, M. Cellular metabolites modulate in vivo signaling of Arabidopsis cryptochrome-1. Plant Signal. Behav. 2015, 10, e1063758. [CrossRef] [PubMed]

26. Jourdan, N.; Martino, C.; El-Esawi, M.; Witczak, J.; Bouchet, P.-E.; d'Harlingue, A.; Ahmad, M. Blue light dependent ROS formation by Arabidopsis Cryptochrome-2 may contribute towards its signaling role. Plant Signal. Behav. 2015, 10, e1042647. [CrossRef] [PubMed]

27. El-Esawi, M.A.; Al-Ghamdi, A.A.; Ali, H.M.; Alayafi, A.A.; Witczak, J.; Ahmad, M. Analysis of Genetic Variation and Enhancement of Salt Tolerance in French Pea (Pisum Sativum L.). Int. J. Mol. Sci. 2018, 19, 2433. [CrossRef] [PubMed]

28. Sallam, A.; Arbaoui, M.; El-Esawi, M.; Abshire, N.; Martsch, R. Identification and Verification of QTL Associated with Frost Tolerance Using Linkage Mapping and GWAS in Winter Faba Bean. Front. Plant Sci. 2016, 7, 1098. [CrossRef] [PubMed]

29. El-Esawi, M.A.; Alaraidh, I.A.; Alsahli, A.A.; Alamri, S.A.; Ali, H.M.; Alayafi, A.A. Bacillus firmus (SW5) augments salt tolerance in soybean (Glycine max L.) by modulating root system architecture, antioxidant defense systems and stress-responsive genes expression. Plant Physiol. Biochem. 2018, 132, 375-384. [CrossRef]

30. Krishnamurthy, S.L.; Sharma, S.K.; Kumar, V.; Tiwari, S.; Singh, N.K. Analysis of genomic region spanning Saltol using SSR markers in rice genotypes showing differential seedlings stage salt tolerance. J. Plant Biochem. Biotechnol. 2016, 25, 331-336. [CrossRef]

31. Kumar, M.; Choi, J.-Y.; Kumari, N.; Pareek, A.; Kim, S.-R. Molecular breeding in Brassica for salt tolerance: Importance of microsatellite (SSR) markers for molecular breeding in Brassica. Front. Plant Sci. 2015, 6, 688. [CrossRef] [PubMed]

32. Kumar, M.; Kim, S.R.; Sharma, P.C.; Pareek, A. Simple and efficient way to detect small polymorphic bands in plants. Genom. Data 2015, 5, 218-222. [CrossRef] [PubMed]

33. Fan, Y.; Shabala, S.; Ma, Y.; Xu, R.; Zhou, M. Using QTL mapping to investigate the relationships between abiotic stress tolerance (drought and salinity) and agronomic and physiological traits. BMC Genom. 2015, 16, 43. [CrossRef] [PubMed]

34. Ahmadi-Ochtapeh, H.; Soltanloo, H.; Ramezanpour, S.S.; Naghavi, M.R.; Nikkhah, H.R.; Yoosefi Rad, S. QTL mapping for salt tolerance in barley at seedling growth stage. Biol. Plant. 2015, 59, 283-290. [CrossRef]

35. Patel, H.K.; Fougat, R.S.; Kumar, S.; Mistry, J.G.; Kumar, M. Detection of genetic variation in Ocimum species using RAPD and ISSR markers. 3 Biotech 2015, 5, 697-707. [CrossRef] [PubMed]

36. Kumar, M. Detection of Genetic Variation in Crop Plants. J. Biomol. Res. Ther. 2015, 4, 2853-2855. [CrossRef]

37. El-Esawi, M.A. Molecular genetic markers for assessing the genetic variation and relationships in Lactuca germplasm. Ann. Res. Rev. Biol. 2015, 8, 1-13. [CrossRef]

38. Malysheva-Otto, L.V.; Ganal, M.W.; Röder, M.S. Analysis of molecular diversity, population structure and linkage disequilibrium in a worldwide survey of cultivated barley germplasm Hordeum vulgare L. BMC Genet. 2006, 7, 6. [CrossRef] [PubMed]

39. Khodayari, H.; Saeidi, H.; Roofigar, A.A.; Rahiminejad, M.R.; Pourkheirandish, M.; Komatsuda, T. Genetic diversity of cultivated barley landraces in Iran measured using microsatellites. Int. J. Biosci. Biochem. Bioinform. 2012, 2, 278-290. [CrossRef]

40. Echart-Almeida, C.; Cavalli-Molina, S. Hordein variation in Brazilian barley varieties Hordeum vulgare L. and wild barley H. euclaston Steud. and H. stenostachys Godr. Genet. Mol. Biol. 2000, 23, 425-433. [CrossRef]

41. Selbach, A.; Cavalli-Molina, S. RAPD characterization of Brazilian barley Hordeum vulgare ssp. vulgare varieties. Euphytica 2000, 111, 127-135. [CrossRef]

42. Oliveira, E.J.; Pádua, J.G.; Zucchi, M.I.; Vencovsky, R.; Vieira, M.L.C. Origin, evolution and genome distribution of microsatellites. Genet. Mol. Biol. 2006, 29, 294-307. [CrossRef]

43. Saghai, M.M.; Biyashev, R.M.; Yang, G.P.; Zhang, Q.; Allard, R.W. Extraordinarily polymorphic microsatellite DNA in barley: Species diversity, chromosomal locations, and population dynamics. Proc. Natl. Acad. Sci. USA 1994, 91, 5466-5470. [CrossRef] 
44. Adawy, S.S.; Saker, M.M.; Haggag, W.M.; El-Itriby, H.A. Amplified Fragment Length Polymorphism (ALFP) based molecular analysis of Egyptian barley lines and landraces differing in their resistance and susceptibility to leaf rust and net blotch diseases. Agri. For. Res. 2008, 58, 125-134.

45. Assefa, A.; Labuschagne, M.T.; Viljoen, C.D. AFLP analysis of genetic relationships between barley (Hordeum vulgare L.) landraces from north Shewa in Ethiopia. Conserv. Genet. 2007, 8, 273-280. [CrossRef]

46. Varshney, R.K.; Chabane, K.; Hendre, P.S.; Aggarwal, R.K.; Graner, A. Comparative assessment of EST-SSR, EST-SNP and AFLP markers for evaluation of genetic diversity and conservation of genetic resources using wild, cultivated and elite barleys. Plant Sci. 2007, 173, 638-649. [CrossRef]

47. Yang, Z.; Chang, Z.; Sun, L.; Yu, J.; Huang, B. Physiological and metabolic effects of 5-aminolevulinic acid for mitigating salinity stress in creeping bentgrass. PLoS ONE 2014, 9, e116283. [CrossRef] [PubMed]

48. Nunkaew, T.; Kantachote, D.; Kanzaki, H.; Nitoda, T.; Ritchie, R.J. Effects of 5-aminolevulinic acid (ALA)-containing supernatants from selected Rhodopseudomonas palustris strains on rice growth under $\mathrm{NaCl}$ stress, with mediating effects on chlorophyll, photosynthetic electron transport and antioxidative enzymes. Electron. J. Biotechnol. 2014, 17, 19-26. [CrossRef]

49. Zhang, Z.-P.; Miao, M.-M.; Wang, C.-L. Effects of ALA on photosynthesis, antioxidant enzyme activity, and gene expression, and regulation of proline accumulation in tomato seedlings under $\mathrm{NaCl}$ stress. J. Plant Growth Regul. 2015, 34, 637-650. [CrossRef]

50. Zhang, C.-P.; Li, Y.-C.; Yuan, F.-G.; Hu, S.-J.; Liu, H.-Y.; He, P. Role of 5-aminolevulinic acid in the salinity stress response of the seeds and seedlings of the medicinal plant Cassia obtusifolia L. Bot. Stud. 2013, 54, 18. [CrossRef] [PubMed]

51. Liu, L.; Nguyen, N.T.; Ueda, A.; Saneoka, H. Effects of 5-aminolevulinic acid on Swiss chard (Beta vulgaris L. subsp. cicla) seedling growth under saline conditions. Plant Growth Regul. 2014, 74, 219-228. [CrossRef]

52. Zhen, A.; Bie, Z.; Huang, Y.; Liu, Z.; Fan, M. Effects of 5-aminolevulinic acid on the H2O2-content and antioxidative enzyme gene expression in NaCl-treated cucumber seedlings. Biol. Plant. 2012, 56, 566-570. [CrossRef]

53. Aksakal, O.; Algur, O.F.; Icoglu Aksakal, F.; Aysin, F. Exogenous 5-aminolevulinic acid alleviates the detrimental effects of UV-B stress on lettuce (Lactuca sativa L.) seedlings. Acta Physiol. Plant. 2017, $39,55$. [CrossRef]

54. Liu, D.; Wu, L.; Naeem, M.S.; Liu, H.; Deng, X.; Xu, L. 5-Aminolevulinic acid enhances photosynthetic gas exchange, chlorophyll fluorescence and antioxidant system in oilseed rape under drought stress. Acta Physiol. Plant. 2013, 35, 2747-2759. [CrossRef]

55. Liu, D.; Hu, L.Y.; Ali, B.; Yang, A.G.; Wan, G.L.; Xu, L. Influence of 5-aminolevulinic acid on photosynthetically related parameters and gene expression in Brassica napus L. under drought stress. Soil Sci. Plant Nutr. 2016, 62, 254-262. [CrossRef]

56. Vos, P.; Mogers, R.M.; Bleeker, M.; Reijans, T.; Vande, L.; Hornes, M.; Frijters, A.; Port, J.; Peleman, J.; Kliper, M.; et al. AFLP: A new technique for DNA fingerprinting. Nucl. Acids Res. 1995, 23, 4407-4417. [CrossRef] [PubMed]

57. Liu, K.J.; Muse, S.V. PowerMarker: An integrated analysis environment for genetic marker analysis. Bioinformatics 2005, 21, 2128-2129. [CrossRef] [PubMed]

58. Peakall, R.; Smouse, P.E. GenAlEx 6.5: Genetic analysis in Excel. Population genetic software for teaching and research-an update. Bioinformatics 2012, 28, 2537-2539. [CrossRef] [PubMed]

59. Nei, M. Estimation of average heterozygosity and genetic distance from a small number of individuals. Genetics 1978, 89, 583-590. [PubMed]

60. Pritchard, J.K.; Stephens, M.; Donnelly, P. Inference of population structure using multilocus genotype data. Genetics 2000, 155, 945-959. [PubMed]

61. Earl, D.A.; Vonholdt, B.M. STRUCTURE HARVESTER: A website and program for visualizing STRUCTURE output and implementing the Evanno method. Conserv. Genet. Resour. 2012, 4, 359-361. [CrossRef]

62. Evanno, G.; Regnaut, S.; Goudet, J. Detecting the number of clusters of individuals using the software STRUCTURE: A simulation study. Mol. Ecol. 2005, 14, 2611-2620. [CrossRef] [PubMed]

63. Lichtenthaler, H.K.; Wellburn, R.R. Determination of total carotenoids and chlorophylls a and b of leaf extracts in different solvents. Biochem. Soc. Trans. 1983, 11, 591-592. [CrossRef]

64. Bradford, M.M. A rapid and sensitive method for the quantification of microgram quantities of protein utilizing the principle of protein dye binding. Anal Biochem. 1976, 72, 248-254. [CrossRef] 
65. Dey, P.M. Oligosaccharides. In Methods in Plant Biochemistry, Carbohydrates; Dey, P.M., Ed.; Academic Press: London, UK, 1990; Volume 2, pp. 189-218.

66. Bates, L.; Waldren, P.P.; Teare, J.D. Rapid determination of free proline of water stress studies. Plant Soil 1973, 39, 205-207. [CrossRef]

67. Velikova, V.; Yordanov, I.; Edreva, A. Oxidative stress and some antioxidant systems in acid rain-treated bean plants: Protective role of exogenous polyamines. Plant Sci. 2000, 151, 59-66. [CrossRef]

68. Heath, R.L.; Packer, L. Photoperoxidation in isolated chloroplasts. I. Kinetics and stoichiometry of fatty acid peroxidation. Arch. Biochem. Biophys. 1968, 125, 189-198. [CrossRef]

69. Zieslin, N.; Ben-Zaken, R. Peroxidase activity and presence of phenolic substances in peduncles of rose flowers. Plant Physiol. Biochem. 1993, 31, 333-339.

70. Zhishen, J.; Mengcheng, T.; Jianming, W. The determination of flavonoid contents in mulberry and their scavenging effects on superoxide radicals. Food Chem. 1999, 64, 555-559. [CrossRef]

71. Ueno, H.; Yamakura, S.; Arastoo, R.S.; Oshima, T.; Kokubo, K. Systematic evaluation and mechanistic investigation of antioxidant activity of fullerenols using $\beta$-carotene bleaching assay. Nanomaterials 2014, 2014,7 .

72. Zhang, J.; Kirkham, M.B. Antioxidant responses to drought in sunflower and sorghum seedlings. New Phytol. 1996, 132, 361-373. [CrossRef] [PubMed]

Sample Availability: Samples of the compounds are not available from the authors. 